\title{
Theoretical optimization of magnetoelectric multilayer laminates
}

\author{
P. Martins ${ }^{1, *}$ C. S. Lehmann Fernandez ${ }^{1}$, S. Lanceros-Méndez ${ }^{2,3}$ \\ ${ }^{1}$ Centro/Departamento de Física, Universidade do Minho, Campus de Gualtar, 4710-057 Braga, Portugal. \\ ${ }^{2}$ BCMaterials, Parque Científico y Tecnológico de Bizkaia, 48160 Derio, Spain \\ ${ }^{3}$ IKERBASQUE, Basque Foundation for Science, 48013 Bilbao, Spain
}

Magnetoelectric (ME) materials are becoming increasingly relevant in the development of new technologies for biomedical applications, sensors and actuators, among others. Mathematical models and simulations allow to optimize features and acquire fundamental knowledge on material properties to achieve innovative developments and devices. In this way, this work is focused on the simulation of both polymer-based and ceramic-based ME laminates, in order to evaluate the influence of their structure, mechanical, electrical and magnetic properties on the ME response.

The effect of size and configuration has been evaluated in Vitrovac/poly(vinylidene fluoride)(PVDF) and Vitrovac/ lead zirconate titanate (PZT) laminated composites. It has been established that the elastic properties and amorphous constitution of PVDF are key parameters governing its ME response, increasing its influence with increasing number of layers in the composite. Although configurations of multi-layers where the laminate is sandwiched between magnetostrictive layers (M-M configurations) exhibited more stable ME response, the structural influence of PVDF in the configurations where the multi-layer is sandwiched between piezoelectric layers (P-P configurations) establishes peaks of ME performance. Good agreement is established when comparing trends reported experimentally in the literature for magnetostrictive to piezoelectric thickness ratio of multilayers, presenting a curve that rapidly increases their $\boldsymbol{\alpha}_{\text {unit }}$ with increasing thickness ratio up to $n=0.3$, when saturation is reached. Further, an optimal configuration for PZT multilayers is found, with external magnetostrictive phases and thickness ratio above 0.2 , leading to a $\mathrm{ME}$ response of $86.7 \mathrm{~V} / \mathrm{cm}$. Finally, it has been established that PVDF configurations with external magnetostrictive phases (M-M configurations) show more stable behaviour and trends over different number of layers, of about $11.5 \mathrm{~V} / \mathrm{cm}$, while P-P configurations present regions with peaks out of the expected trend with ME response (48 $\mathrm{V} / \mathrm{cm}$ )closer to the one of ceramic multilayers .

*corresponding authors: senentxu.lanceros@bcmaterials.net; pmartins@ fisica.uminho.pt

Keywords: magnetoelectric, piezoelectric, magnetostrictive, finite elements method, laminates 


\section{Introduction}

Magnetoelectric (ME) and multiferroic materials are being increasingly studied from the fundamental and application point of view. The coupling between magnetic and electric properties allows to tackle novel scientific challenges and the development of multifunctional devices if the magnetic and electrical orders can be mutually controlled [1]. Further, the miniaturization of devices has guided the attention into a new generation of multifunctional materials, that allow a wide range of applications from broadband magnetic field sensors, actuators and memory devices, to magnetically controlled electro-optic or piezoelectric devices, among others [2, 3].

Single-phase materials with ferromagnetic and ferroelectric properties include XXX, but are limited in number [4] and present low ME performance and low Curie temperature[5]. Thus, efforts have been established to develop composites with higher ME coupling than single-phase materials[6]. Composites with magnetostrictive (ms) and piezoelectric (pzo) phases provide the best systems to improve ME response, and the knowledge of these materials from the fundamental point of view is the key tool to achieve optimized performances, being theoretical models and simulations a key element to optimize coupling and geometry $[7,8]$.

The ME coupling may arise by the effect of an electric (magnetic) field on the magnetization (polarization) of a material, or indirectly via strain over the magnetostrictive-electroactive domains of a ME composite [4]. The ME voltage coefficient $\left(\alpha_{M E}\right)$ is described as the change in electrical polarization, $P$, caused by the variation of the magnetic field, $H$, as[9]:

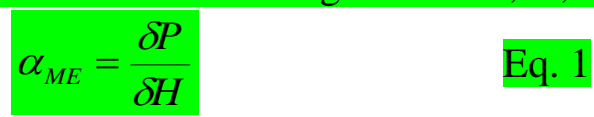

The ME effect in composites is more than 2 orders higher than for single-phase materials[10] and can be found in magnetic metals/alloys and piezoelectric ceramic composites, laminated metals/alloys and piezoelectric polymers and particulate composites of ferrite and piezoelectric ceramics[5].

Ceramic ME composites exhibit much higher $\alpha_{M E}$ than polymer-based composites, but the latter have the advantage of a good strain coupling and durability, higher flexibility, without leakage currents, the possibility of being shaped into a variety of forms (sheets, moulded shapes), and the low temperature processing [5, 11]. Further, some of them are biocompatible [12]. Further, polymer-based composites avoid the limitations of the interface reactions on the ceramic composites that cause high dielectric losses and may affect the stability of the developed ME devices[5].

Polymer-based ME composites exist as laminates or nanocomposites (low dimensionalstructured). Although low-dimensional structured composites present no interface degradation, laminate types of ME materials present a ME coefficient that can reach up to four orders of magnitude higher than the ones reported on nanocomposites [13]. ME composites with a laminated bi-layer or multi-layer configuration show also advantages such as avoiding the polarization loss in bulk composites[14]. The piezoelectric phase can be poled to enhance the ME coupling and it is also possible to change the poling and applied field directions to achieve maximum ME coupling[15]. Mathematical models and, particularly, Finite element method simulation models 
represent a suitable tool to optimize the performance of such new materials [7, 8], by incorporating a wide spectrum of parameters, including physico-chemical characteristics of materials and geometries [7, 8]. This methodology is even more interesting when the ME performance of the materials are difficult to obtain by direct measurements and provide knowledge that can lead to the production of new and optimized materials for devices[16].

Previous works have been devoted to study the ME effect at the interface between the magnetostrictive and piezoelectric phases in low dimensional polymer based fibrilar [7] and spherical structures[8]. Similar mathematical models and simulations were used in order to study the ME effect at the interface between the magnetostrictive and piezoelectric phases.

Thus, this work reports on the multilayer structure optimization of ceramic-based and polymer-based composites, taking into consideration the effects of $\mathrm{xxxxx}$, allowing not just the understating of the coupling between magnetostrictive and piezoelelctric layers and their dependence on material characteristics, but also the design of optimized structures for device applications.

For the simulations, piezoelectric poly(vinylidene fluoride) (PVDF) was the selected polymer due to its high piezoelectric response, thermal, mechanical and chemical resistance[15, 17]. The piezoelectric ceramic material was $\mathrm{PZT}^{\prime}-5 \mathrm{H}$, based on its piezoelectric response and complete characterization [18-24]. Vitrovac 4040®[25] $\left(\mathrm{Fe}_{39} \mathrm{Ni}_{39} \mathrm{Mo}_{4} \mathrm{Si}_{6} \mathrm{~B}_{12}\right)$ was used as magnetostrictive component based on its high piezomagnetic coefficient at low magnetic fields [16].

\section{Theoretical Models}

\subsection{Electromechanical finite element method model for ME laminates}

As both magnetostrictive and piezoelectric behaviors are anisotropic and as the model incorporates specific mechanical coupling factors into the final ME response, finite element method (FEM) is a suitable approach for the theoretical stidy of laminated ME composites [16]. Assuming a stresses state on the magnetostrictive and piezoelectric phases, and linear range of magnetostriction, the electro-mechanical coupling was modeled following the main guidelines reported in references [7, 8, 16] and considering perfect bonding between interphases. Additionally, a 2D model was assumed considering the ME effect to be constant along the width of the structure. Based on the constitutive equations [26] the calculations were performed on each nodal point with specific boundary conditions and holding into two essential principles: energy conservation and continuity at the nodal interface. The electromechanical simulation consisted in applying a deformation on the lateral ends of the magnetostrictive layer taking into account the magnetostrictive response of the material $[7,8,16,25,27]$ and evaluating the electric potential developed across the piezoelectric layer. The input parameter for the calculations was $S=\lambda(H)$, which is the magnetically induced magnetostrictive strain, the magnetostriction curve of the material. In all cases, it was selected the strain corresponding to the maximum deformation experienced by the magnetostrictive layer. Structurally, as the layers were perfectly bonded and 
assuming linear range of magnetostriction, the deformation on the magnetostrictive layer produces a deformation on the other layers that will depend on their mechanical properties. Boundary conditions as lateral polarization of the piezoelectric material, grounds, and fixing the ME structure only to deform along the longitudinal direction were also set.

\subsection{Mechanical model for the piezoelectric material}

Constituent equations for the linear piezoelectric effect (in stress charge form) are given by the direct and indirect piezoelectric effects [28, 29], respectively:

$$
\begin{array}{ll}
\sigma_{m}=c_{E} \cdot S-e_{S} \cdot E & \text { Eq. } 2 \\
D=e_{s}^{T} \cdot S+\varepsilon_{s} \cdot E & \text { Eq. } 3
\end{array}
$$

where $\sigma_{m}$ is the stress vector, $D$ is the electric flux density vector, $S$ is the strain vector, $E$ is the electric field vector, $c_{E}$ is the elasticity matrix (evaluated at constant electric field) and $\varepsilon_{s}$ is the dielectric matrix (evaluated at constant mechanical strain).

The mechanical model for magnetostrictive material consists in a linear elastic model, fulfilled by the continuity equations on stationary case when the phase is subjected to maximal magnetostriction $[7,8,16,30]$ :

$$
\begin{aligned}
& \quad \nabla \cdot D=\rho_{V} \quad \text { (Gauss Law, Eq. } 4 \\
& \begin{array}{l}
\mathrm{t}=0) \\
\nabla \cdot \sigma=f_{V} \quad \text { (Cauchy momentum equation, Eq. } 5
\end{array}
\end{aligned}
$$

where the divergence of the electrical displacement field, D, gives the free electric charge density, $\rho_{V}$, and the divergence of the stress tensor $\sigma$, gives the force per unit volume, $f_{V}$. The model also considers traction-free boundary conditions at outer surfaces and perfect bonding at all the interfaces of the structure (continuity equations of displacement and normal stress).

In order to obtain the electric potential $(\varphi)$, the assumption of an electrode on the external surface of the ME structure is made, as an electrically floating electrode condition (where zero charge is held), therefore given by:

$$
E=-\nabla \varphi \quad \text { Eq. } 6
$$

where $E$ is the local electric field strength.

The obtained values for electric potential distribution were integrated over the electric potential interphase line, and $\alpha_{M E}$ is defined as (equation 7): 


$$
\alpha_{M E}=\left|\frac{d E}{d H}\right|=\left|\frac{\delta V}{d_{p z o}}\right|=\left|\frac{\int_{\text {int erface }} \varphi \cdot d L}{d_{p z o}(\mathrm{~cm}) \cdot L}\right|\left(\frac{V}{\mathrm{~cm}}\right) \quad \text { Eq. } 7
$$

where $\delta V$ is the induced ME voltage, $d_{p z o}$ is the piezoelectric thickness (in centimeters), $\varphi$ is the electric potential, and $L$ is the interface length.

In cases of experiments with more than one piezoelectric layer, the composite has to be treated as a device where piezoelectric layers are connected in series. The final ME coefficient, $\alpha_{M E}$, is obtained by the sum of each ME coefficient, over each interface, as:

$$
\alpha_{M E}=\sum_{I N T E R F A C E} \alpha_{M E-I N T E R F A C E} \quad \text { Eq. } 8
$$

In other multilayer experiments, a unitary ME coefficient, called unitary $\alpha$, or $\boldsymbol{\alpha}_{\boldsymbol{u} \text { unit }}$, can be defined by the sum of all partial electric potentials between magnetostrictive and piezoelectric layers, divided by the total piezoelectric length (in $\mathrm{cm}$ ), as:

$$
\alpha_{\text {unit }}=\left|\frac{d E}{d H}\right|=\left|\frac{\delta V}{d_{p z o}}\right|=\frac{\sum_{\text {INTERFACE }}\left|\int_{\text {int erface }} \varphi \cdot d L\right|_{\text {INTERFACE }}}{d_{p z o}(\mathrm{~cm}) \cdot L}\left(\frac{V}{\mathrm{~cm}}\right) \quad \text { Eq. } 9
$$

where $\boldsymbol{\alpha}_{\text {unit }}$ is the unitary ME coefficient for the multilayer, $\boldsymbol{\delta} \boldsymbol{V}$ is the induced ME voltage, $\boldsymbol{d}_{\boldsymbol{p} z o}$ is the total piezoelectric thickness (sum of all individual piezoelectric thicknesses, in $\mathrm{cm}$ ), $\varphi$ is the electric potential and $\boldsymbol{L}$ is the interface length. For symmetric cases, $\boldsymbol{\alpha}_{\boldsymbol{u} \text { it }}$ corresponds also to the average $\boldsymbol{\alpha}_{\boldsymbol{M E}}$, or the sum of all $\alpha$-values divided by the number of piezoelectric layers.

\subsection{Multilayer ME laminate model experiments}

ME performance of bilayered and multilayered laminates has been studied for piezoelectric ceramics and magnetostrictive metals [21, 22, 31, 32], but variations on the mechanical properties in the piezoelectric component (when including a piezoelectric polymer as PVDF, more flexible and with an amorphous semi-crystalline structure) may lead to variations in the ME performance of the composite. Finite element Method (FEM) simulation analyses were performed in order to evaluate the influence of the structural amorphous semicrystalline properties of PVDF on the ME performance of the ME laminates and to compare them with the results obtained for a ceramic piezoelectric material, as lead zirconate titanate (PZT-5H; Pb[ZrxTi1-x]O3; from now on PZT). A two-dimensional approximation was considered, establishing that the ME response of the structure will be constant along the width of the structure. Vitrovac $4040 \AA$ was set to be the magnetostrictive part in the ME composite for both piezoelectric cases.

Piezoelectric calculations are based on linear models, as described by the constitutive equations, with the boundary condition of electrical ground in one of the surfaces or interphases in front of the other electrically isolated surface (interphase) where the electric potential was 
evaluated. The applied deformation of the magnetostrictive material produces a deformation on the piezoelectric domain generating indirectly via strain an electric potential distribution on the interface between the piezoelectric and magnetostrictive material.

For all purposes, the length of the ME structure was set to be $30 \mathrm{~mm}$ with an increase of 1 $\mathrm{mm}$ at each end of each piezoelectric layer, with a width of $6 \mathrm{~mm}$. The piezoelectric material polarization is established to be lateral. Simulations assumed maximal deformation for the magnetostrictive material, obtaining the structural piezoelectric response for the piezoelectric ceramic and the piezoelectric polymer when the magnetostrictive layer is deformed to its maximal value from the magnetostriction curve. This allowed to establish the influence of the mechanical properties and the influence of the morphology of the materials on the ME performance of different laminate configurations.

\subsection{Magnetostrictive and piezoelectric material properties}

The selected magnetostrictive component, Vitrovac 4040® [25], shows a modest magnetostriction of $\lambda \max =8 \mathrm{ppm}$, and a high piezomagnetic coefficient of $1.3 \mathrm{ppm} / \mathrm{Oe}$ at low magnetic fields $(\approx 15$ Oe) [16]. The maximum magnetostriction for the applied deformation is obtained from the magnetic field-magnetostriction curve of the material[25].

For the piezoelectric component, PVDF was selected since it is the overall best piezoelectric polymer [5, 15]. Lead zirconate titanate PZT was selected as it is also among the most used piezoelectric ceramics.

The main properties of the different materials used in for the simulations are presented in ¡Error! No se encuentra el origen de la referencia. .

Table 1: Structural and magnetostrictive properties of Vitrovac4040[25] and relevant electrical and mechanical properties of PVDF[33] and PZT-5H[16].

\begin{tabular}{|c|c|c|c|c|c|c|}
\hline \multicolumn{4}{|c|}{ Magnetostrictive Material Property } & \multicolumn{2}{|c|}{ Vitrovac4040 } & \\
\hline \multirow{3}{*}{\multicolumn{2}{|c|}{ Mechanical }} & \multicolumn{2}{|c|}{ Density } & $\rho$ & $\left(\mathrm{kg} / \mathrm{m}^{3}\right)$ & 7900 \\
\hline & & \multicolumn{2}{|c|}{ Young modulus } & $\mathbf{Y}$ & $(\mathrm{GPa})$ & 1.5 \\
\hline & & \multicolumn{2}{|c|}{ Poisson's ratio } & ? & 1 & 0.27 \\
\hline \multirow{3}{*}{\multicolumn{2}{|c|}{ Magnetostrictive }} & \multicolumn{2}{|c|}{ Magnetostriction } & $\lambda_{s}$ & $\mathrm{ppm}$ & 8 \\
\hline & & \multirow{2}{*}{\multicolumn{2}{|c|}{$\begin{array}{c}\text { Piezomagnetic c. } \\
\text { Magnetic field }\end{array}$}} & $k$ & $\mathrm{ppm} / \mathrm{Oe}$ & 1.3 \\
\hline & & & & $H$ & Oe & $\approx 15$ \\
\hline \multicolumn{4}{|c|}{ Piezoelectric Material Property } & \multicolumn{2}{|c|}{ PVDF } & PZT-5H \\
\hline \multirow{3}{*}{$\begin{array}{l}\text { Mechanic } \\
\text { al }\end{array}$} & Density & $\rho$ & $\left(\mathrm{kg} / \mathrm{m}^{3}\right)$ & \multicolumn{2}{|c|}{1470} & 7500 \\
\hline & $\begin{array}{l}\text { Elasticity } \\
\text { Matrix }\end{array}$ & & (GPa) & $\begin{array}{r}\{\{2.74, \\
\{5.21 \mathrm{e} \\
\{4.78 \\
\{0, \\
\{0, \\
\{0,0\end{array}$ & $\begin{array}{l}78,0,0,0\} \\
21 e, 0,0,0\} \\
12,0,0,0\} \\
4,0,0\} \\
2.74,0\} \\
2.74\}\}\end{array}$ & $\begin{array}{c}\{11.27 \mathrm{E}+11,8.02 \mathrm{E}+10,8.47 \mathrm{E}+10,0,0,0\} \\
\{8.02 \mathrm{E}+10,1.27 \mathrm{E}+11,8.47 \mathrm{E}+10,0,0,0\} \\
\{8.47 \mathrm{E}+10,8.47 \mathrm{E}+10,1.17 \mathrm{E}+11,0,0,0\}, \\
\{0,0,0,2.30 \mathrm{E}+10,0,0\} \\
\quad\{0,0,0,0,2.30 \mathrm{E}+10,0\} \\
\{0,0,0,0,0,2.35 \mathrm{E}+10\}\}\end{array}$ \\
\hline & $\begin{array}{c}\text { Compliance } \\
\text { Matrix }\end{array}$ & $C_{E}^{-1}$ & $(1 / \mathrm{GPa})$ & $\begin{array}{l}\{0.365,-C \\
-0.192,0 .\end{array}$ & $\begin{array}{l}424,-0.209 \\
, 0,0.365,0\end{array}$ & $\begin{array}{c}\{1.65 \mathrm{e}-11,-4.78 \mathrm{e}-12,1.65 \mathrm{e}-11,-8.45 \mathrm{e}-12 \\
-8.45 \mathrm{e}-12,2.07 \mathrm{e}-11,0,0,0,4.35 \mathrm{e}-11,0 \\
0,0,0,4.35 \mathrm{e}-11,0,0,0,0,0,4.26 \mathrm{e}-11\}\end{array}$ \\
\hline
\end{tabular}




\begin{tabular}{|c|c|c|c|c|c|}
\hline & & & & $\begin{array}{c}0,0,0,0.365,0,0,0,0,0 \\
0.365\}\end{array}$ & \\
\hline \multirow{2}{*}{ Electrical } & $\begin{array}{l}\text { Coupling } \\
\text { Matrix }\end{array}$ & e & $\left(\mathrm{C} / \mathrm{m}^{2}\right)$ & $\begin{array}{c}\{\{0,0,-4.761,0,0,-33.33\} \\
\{0,0,3.703,0,1.703,0\} \\
\{1.703,0,0,0,0,0\}\}\end{array}$ & $\begin{array}{c}\{\{0,0,-6.62281,0,0,-6.62281\}, \\
\{0,0,23.2403,0,17.0345,0\}, \\
\{17.0345,0,0,0,0,0\}\}\end{array}$ \\
\hline & $\begin{array}{c}\text { Relative } \\
\text { Permittivity }\end{array}$ & $\varepsilon_{\mathrm{s}}$ & 1 & $\begin{array}{l}\{\{13,0,0\} \\
\{0,13,0\} \\
\{0,0,13\}\}\end{array}$ & $\begin{array}{l}\{\{1704.4,0,0\} \\
\{0,1704.4,0\} \\
\{0,0,1433.6\}\}\end{array}$ \\
\hline
\end{tabular}

\subsection{Multi-layer configurations}

Biphasic, triphasic and multiphasic ideal models (with perfectly bonded interphases) were modeled with different configurations and thicknesses for the two different piezoelectric materials (PVDF and PZT). The modelled configurations were classified according to their piezoelectric material, number of layers, and the type of external layer, into:

- M-P multi-layers: in this configuration the composite has an even number of layers with one magnetostrictive and one piezoelectric layer at the ends. The considered number of layers was 2, 4, 6, 8 and 10. The simplest case presented is the bi-layer PM configuration.

- M-M multi-layers: with an odd number of layers, the composite is always sandwiched between two magnetostrictive layers. The considered number of layers was 3, 5, 7 and 9 . The simplest case presented is the trilayer MPM configuration.

- P-P multi-layers: with an odd number of layers, the composite is sandwiched between two piezoelectric layers. The considered number of layers was 3, 5, 7 and 11 . The simplest case is the PMP trilayer configuration.

\subsection{Independent thickness sweep experiment}

First, independent magnetostrictive and piezoelectric thicknesses sweep were carried on. For the bilayer experiment, magnetostrictive and piezoelectric thickness were swept independently between $1 \mu \mathrm{m}$ and $1 \mathrm{~mm}$. For both M-M and P-P configurations, the sweep included values for thickness between $10 \mu \mathrm{m}$ and $600 \mu \mathrm{m}$. In all cases, when the magnetostrictive layer thickness was swept, the piezoelectric material thickness was held at a constant value of $110 \mu \mathrm{m}$. Similarly, when the piezoelectric material thickness was varied, the magnetostrictive material thickness was set at a constant value of $25 \mu \mathrm{m}$. For all cases, free and clamped configurations were analyzed. In the clamped configurations, the laminated structure was set to deform only along longitudinal direction in both bottom and top piezoelectric surfaces, similar to material fixed into a devices.

When more than one piezoelectric layer is included, the ME coefficient was obtained by equation 7, applied in each interface and then treated as a device where both piezoelectric layers are connected in series. Therefore, the final ME coefficient $\left(\boldsymbol{\alpha}_{M E}\right)$ was obtained by the sum of each 
ME coefficient, over each interface, as presented in equation 8. Then, the unitary ME coefficient, $\alpha_{\text {unit, }}$ was also calculated by equation after 9 .

Figure 1 presents a schematic illustration of the simplest configuration of each experimental group, M-P, M-M and P-P.
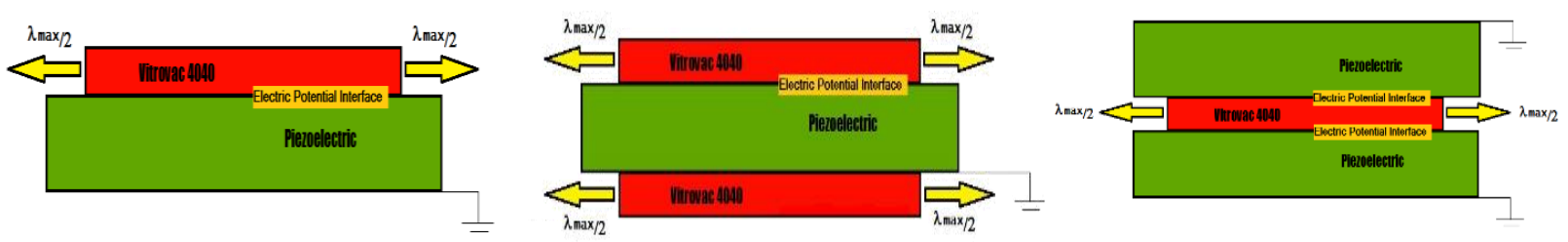

Figure 1. Representative illustration of each type of simulation experiments, with the lowest considered number of layers. M-P bilayer on the left, MPM (M-M trilayer) configuration on the middle and PMP (P-P trilayer) configuration on the right.

In order to corroborate the trends obtained in the simulations, results were post-processed in order to be compared with data from the literature. For bilayers, Ryu et al. [22] reported a constant ME coefficient for higher Terfenol-D/PZT thickness ratios, that decays for thicknesses ratios below 10, establishing values for $0.5,0.4$ and $0.3 \mathrm{~mm}$ of PZT. On the case of MPM trilayers, Ryu et al. [23] established the theoretical expectation for the $\mathrm{ME}$ voltage coefficient $(\mathrm{dE} / \mathrm{dH})$ as function of the thickness ratio (d_ms/d_pzo) between Terfenol-D and PZT, showing that the output voltage increases while increasing the thickness ratio, saturating above d_ms/d_pzo $=10$. Peak values of $\boldsymbol{\alpha}_{M E}$ as function of $\mathrm{n}$-ratio $\left(2 * \mathrm{~d} \_\mathrm{ms} /\left(2 * \mathrm{~d} \_\mathrm{ms}+\mathrm{d} \_\mathrm{pzo}\right)\right)$ were also reported for Metglas/PZT/Metglas [18] . These results were also confirmed experimentally for Terfenol-D/PZT/Terfenol-D laminates [23], for Co/PZT/Co heterostructures ,. [34], and for the ME performance of different thickness ratio values of Terfenol-D/PMN-PT/Terfenol-D laminates [35].

\subsection{Constant ME thickness multilayer experiment}

In this case, the simulation experiments consisted in dividing a total thickness of $D_{c}=300 \mu \mathrm{m}$ and $D_{c}=600 \mu \mathrm{m}$ by the total number of layers of the composite. For example, for a number of layers $\mathrm{N}=4$, each layer would be $75 \mu \mathrm{m}$ thick for the $300 \mu \mathrm{m}$ composite and $150 \mu \mathrm{m}$ for the 600 $\mu \mathrm{m}$ composite. In order to assess the limitations of this structural analysis, results were compared with those obtained by Srinivasan [36, 37], that experimentally analyzed a $400 \mu \mathrm{m}$ thick bilayer and multilayer thick-film structures of $\mathrm{La}_{0.7} \mathrm{Sr}_{0.3} \mathrm{MnO}_{3}$ (LSMO)-PZT and $\mathrm{La}_{0.7} \mathrm{Ca}_{0.3} \mathrm{MnO}_{3}(\mathrm{LCMO})$ PZT. Those samples were fabricated with an equal number of manganite and PZT layers, between 2 and 8. The unitary ME coefficient was obtained from equation 9. The even-numbered layer experiment is schematically represented in Figure 2. 

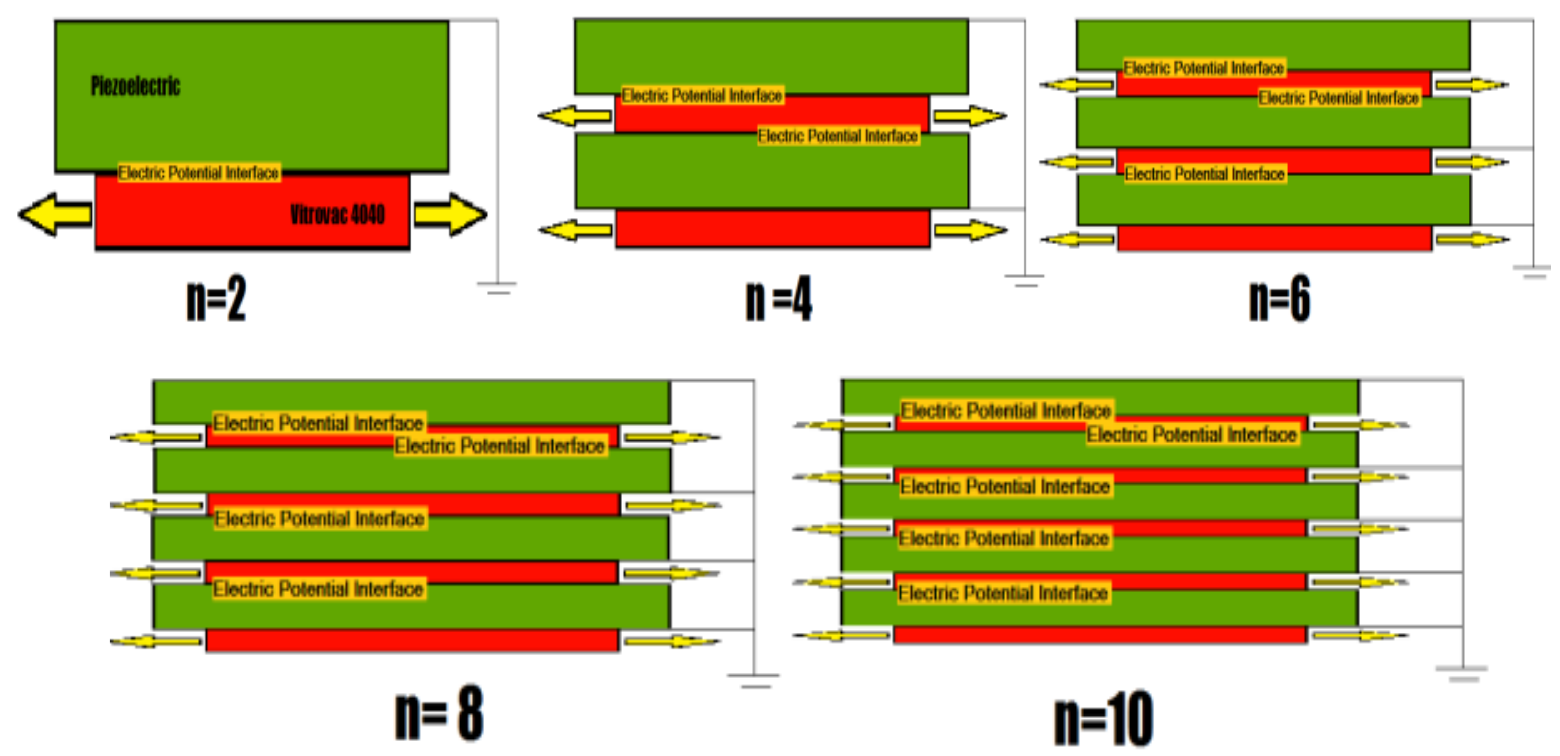

Figure 2: Schematic representation of M-P Multilayer ME simulation experiments, where $n$ is the number of layers and the total thickness $D_{c}$ was set to $300 \mu \mathrm{m}$ and $600 \mu \mathrm{m}$.

\section{Results and Discussion}

\subsection{Bi-layered composites}

Figure 3 shows the simulated ME coefficient, $\alpha_{M E}$, of the bilayer laminate structure for both PVDF and PZT as piezoelectric layers, including magnetostrictive (d_ms) and piezoelectric (d_pzo) thickness sweeps from $10 \mu \mathrm{m}$ to $1 \mathrm{~mm}$. The electric potential distribution of two particular simulations is also represented to illustrate the effect of the type of piezoelectric material.

b)

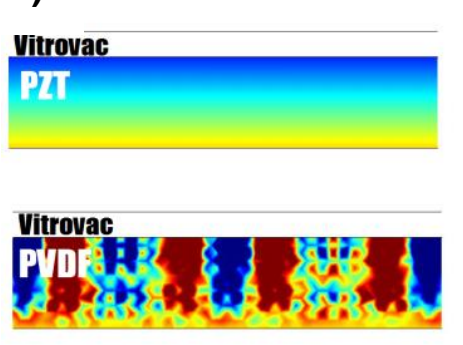




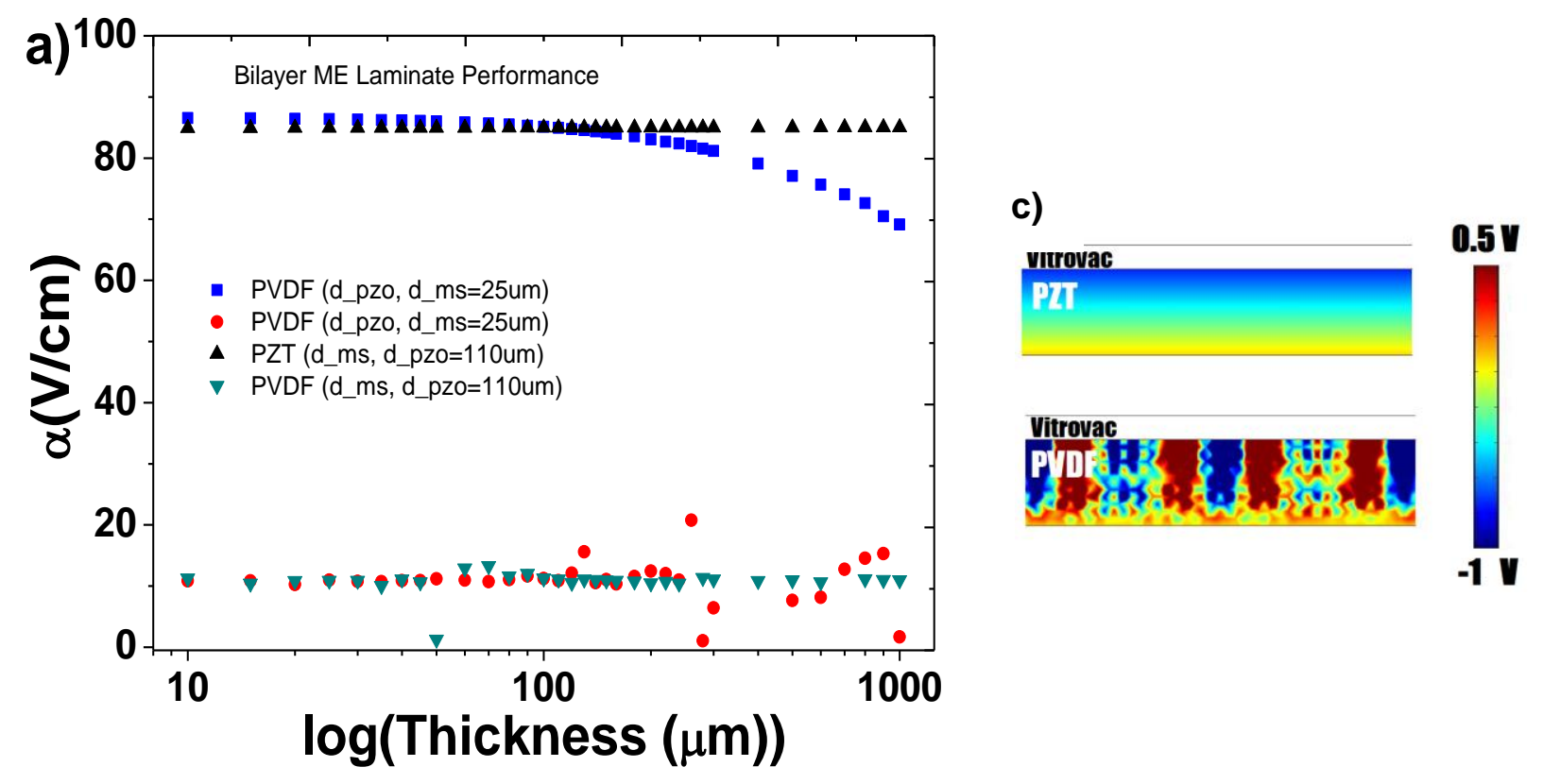

Figure 3: Bilayered ideal laminate simulation results as a function of the magnetostrictive (d_ms) and piezoelectric (d_pzo) thickness. Figure (a) shows the ME coefficient, $\alpha(\mathrm{V} / \mathrm{cm})$, of composites with constant piezoelectric thickness of (d_pzo) $110 \mu \mathrm{m}$, for different magnetostrictive material thicknesses (d_ms) and with constant d_ms $=25 \mu \mathrm{m}$, for different piezoelectric thicknesses (d_pzo). Figure (b) and (c) shows the electric potential distribution for bilayered ME laminates with the following dimensions (b) d_ms $=90 \mu \mathrm{m}, \mathrm{d} \_p z o=110 \mu \mathrm{m}$ (c) d_ms $=25 \mu \mathrm{m}, \mathrm{d} \_\mathrm{pzo}=90 \mu \mathrm{m}$, for both piezoelectric PVDF and PZT.

By establishing magnetostriction as a deformation, it may be expected the independence of the magnetostrictive layer thickness on the ME performance of the composite, which is confirmed by the results obtained for the magnetostrictive thickness sweep presented in Figure 3 (a) for bilayer composites. Small irregularities are introduced by the semicrystalline nature of PVDF in the ME response curves for magnetostrictive thickness sweep, resulting in above average $\alpha$ value for Vitrovac thicknesses of $20 \mu \mathrm{m}$ and $300 \mu \mathrm{m}$, and below average $\alpha$ values for magnetostrictive thicknesses of $50 \mu \mathrm{m}$ and $700 \mu \mathrm{m}$. For PZT thickness sweep (Figure xxx), it is obtained a constant ME coefficient for higher d_ms/d_pzo thickness ratio, that decays for thicknesses ratios below 10, following the trends presented by Ryu et al. [22]. For PVDF-thicknesses below $100 \mu \mathrm{m}$ also a constant ME response is obtained. On the other hand, irregular values are obtained for the ME response of samples with piezoelectric layer thickness above $200 \mu \mathrm{m}$, as shown in the Figure 3 (a).

Figure 3 (b) and (c) show the electric potential distribution for two cases of PVDF and PZT, revealing a strong influence of the PVDF's semi-crystalline constitution in the electrical properties. While the piezoceramic establishes a uniform laminar pattern of electric potential distribution in the polarization direction, the formation of clusters of heterogeneous regions with an irregular electric potential distribution is observed in the PVDF's simulation result. Therefore, this represents one of the main differences between the piezoelectric ceramic and the polymer, related to the irregular ordering in which dipoles generate the piezoelectric response in the PVDF's 
samples, based on the random distribution of the crystalline phase, main responsible for the piezoelectric response, along the sample.

On average, for constant piezoelectric thicknesses, it is observed that the performance of the PVDF bilayered composite is about $\approx 12 \%$ the one of the PZT counterpart. For equal piezoelectric thicknesses below $200 \mu \mathrm{m}$ and constant magnetostrictive thickness of d_ms=25 $\mu \mathrm{m}$, the PVDF composite shows an average $\alpha$ value of $\approx 8 \%$ the one of the PZT counterpart.

\subsection{Trilayered composites}

Trilayered ME laminates may be composed of one piezoelectric layer sandwiched between two magnetostrictive layers (MPM configuration) or by one magnetostrictive layer intercalated between two piezoelectric layers (PMP configuration). When two piezoelectric layers are included, two sources of electric potential are involved which require considering the ME response $\alpha$-values by equation ( 7 and 8 ). When configurations are to be compared, it is desirable to establish one single value that provides information on the performance of the material per unit piezoelectric length, which is obtained by equation (9), defining the unitary ME coefficient, $\boldsymbol{\alpha}_{\text {unit }}$.

Figure 4 and Figure 5 display the ME response of free and clamped, MPM and PMP ME laminates as compared to the bilayer configuration for magnetostrictive and piezoelectric thicknesses sweep, respectively, for each trilayered composite with PZT (a) and PVDF (b) as piezoelectric component. For the magnetostrictive sweep (Figure 4) of PZT trilayers, it is observed that the addition of one extra layer of magnetostrictive material (MPM) results in an increase of about $1 \mathrm{~V} / \mathrm{cmOe}$ in the ME coefficient for all simulated magnetostrictive thickness range, when compared to the bilayer structure. It is also verified that the addition of an extra piezoelectric layer (PMP configuration) allows to double the ME response (by treating the composite as a single device and obtaining $\boldsymbol{\alpha}_{\boldsymbol{M E}}$ by eq. 9). The independence of $\boldsymbol{\alpha}_{\boldsymbol{M E}}$ over the magnetostrictive thickness remains generally valid for all configurations, with the only exceptions of specific PVDF configurations, in which the semicrystalline nature of PVDF plays a key role in establishing isolated peaks of ME response. 

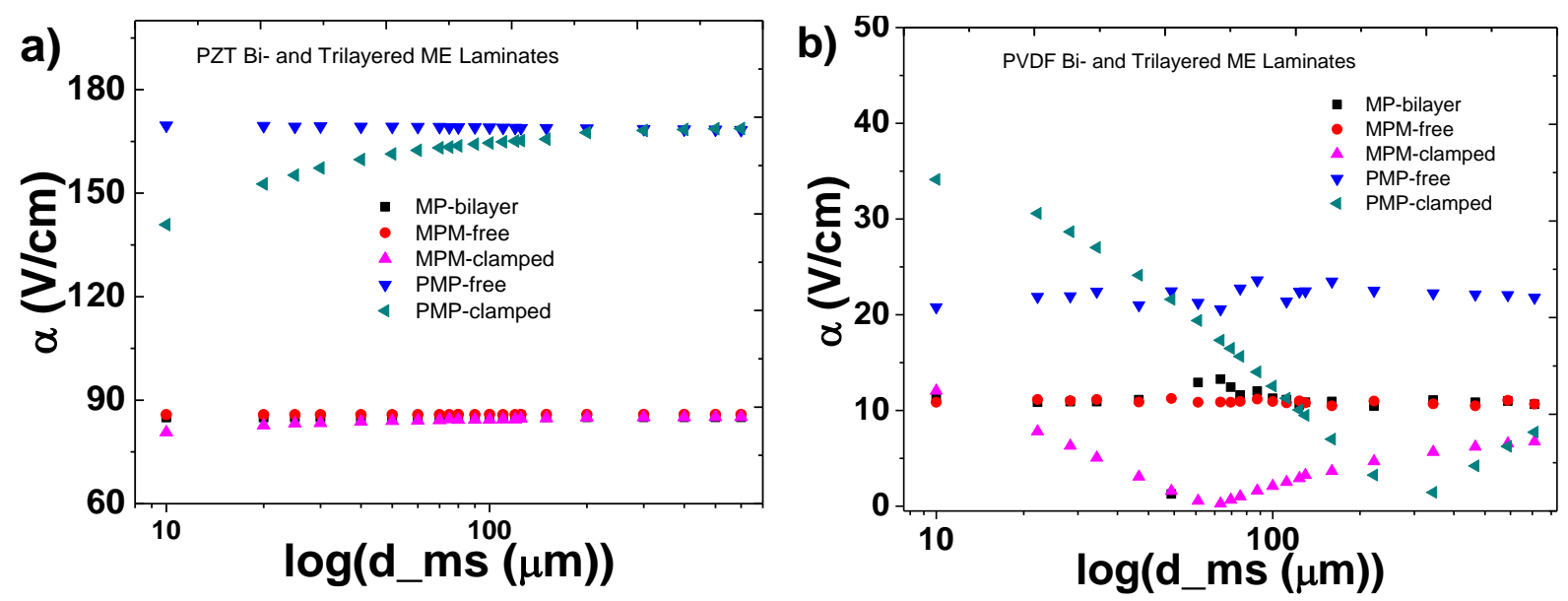

Figure 4: Simulation results of the ME coefficient of bilayered and trilayered ideal laminate composites as a function of the the magnetostrictive layer thicknesses (d_ms). Figure (a) shows the ME response (ME coefficient, $\alpha(\mathrm{V} / \mathrm{cm})$ ) of free and clamped, MPM and PMP ME structures with PZT as piezoelectric layer. Figure (b) shows the ME response (ME coefficient, $\alpha(\mathrm{V} / \mathrm{cm})$ ) of free and clamped, MPM and PMP ME structures with PVDF as piezoelectric material.

For MPM trilayered configurations with PZT as piezoelectric layer, clamping decreases the ME response progressively with decreasing magnetostrictive layer thicknesses (d_ms) below $150 \mu \mathrm{m}$, after which presented same order of $\alpha$ of the free trilayered structure. For clamped PVDF trilayer composites, these differences are even larger, leading to nearly zero ME response for magnetostrictive thickness of $70 \mu \mathrm{m}$ for the MPM configuration. As PVDF is a viscoelastic material, and there is no structural damping considered, simulation results of the bilayer and the MPM-free configuration do not revealed relevant differences. The extra magnetostrictive layer has the effect of comprising (fixing, homogenizing) the ME coefficient to $\approx 11 \mathrm{~V} / \mathrm{cm}$, the same value as for the thinnest and thickest magnetostrictive thicknesses values in the bilayer configuration. For the same reason, the behaviour of the PVDF-PMP-clamped configuration for low thicknesses of Vitrovac surpassed the standard improvement of duplicating the ME performance of the bilayer (triplicating the $\alpha_{\mathrm{MEe}}$ of the bilayer), decreasing $\alpha$-values for higher magnetostrictive thicknesses. The lower performance was obtained for the particular case of Vitrovac with a thickness of 200 $\mu \mathrm{m}$, the ME response increasing with increasing magnetostrictive layers thickness. For thinner magnetostrictive thickneses, the PVDF clamped configuration show higher $\alpha$-values than the free one, decreasing the performance for higher magnetostrictive thickness layers $\mathrm{s}$ and matching the performance of the free configuration for magnetostrictive thickness of $50 \mu \mathrm{m}$ and later the bilayer performance for d_ms $=100 \mu \mathrm{m}$. This allows to conclude that clamping would only be useful for thickness below d_ms $=50 \mu \mathrm{m}$. 

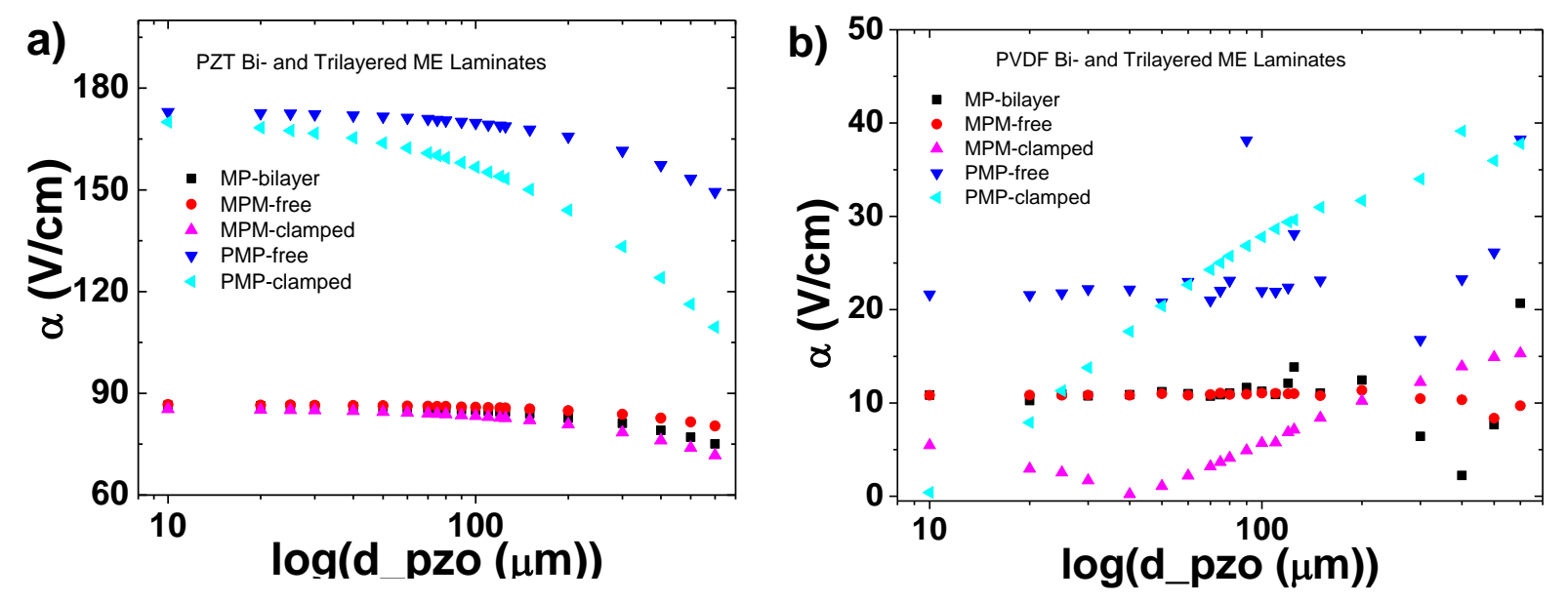

Figure 5: Bilayered and trilayered ideal laminate magnetoelectric coefficient as a function of the magnetostrictive layer thicknes (d_pzo). Figure (a) shows the ME response (ME coefficient, $\alpha$ $(\mathrm{V} / \mathrm{cm})$ ) of free and clamped, MPM and PMP ME structures of PZT. Figure (b) shows the ME response (ME coefficient, $\alpha(\mathrm{V} / \mathrm{cm})$ ) of free and clamped, MPM and PMP ME structures of PVDF.

As shown in Figure 5, all PZT configurations present a constant $\boldsymbol{\alpha}_{M E}$ value when the piezoelectric thickness is below $60 \mu \mathrm{m}$, decreasing for thicker piezoelectric thicknesses. Although the general trends for the three configurations remain similar, the clamped configuration exibits the lowest ME performance, followed by the bilayered one, showing the free configuration the highest ME performance.

PVDF bilayer- and MPM free-configurations results displayed similar ME performance in the range of piezoelectric thickness below $200 \mu \mathrm{m}$, establishing that the free configuration shows a more stable trend than the bilayered one. The free configuration show a stable $\boldsymbol{\alpha}_{M E}$ of approximately $11 \mathrm{~V} / \mathrm{cm}$ for the range of piezoelectric thickness below $200 \mu \mathrm{m}$, decreasing with increasing piezoelectric thicknesses above $200 \mu \mathrm{m}$. The clamped configuration shows a decaying value of $\boldsymbol{\alpha}_{M E}$ for piezoelectric PVDF thickness in the range 10 to $40 \mu \mathrm{m}$, increasing for thicker PVDF layers, obtaining better ME performance than the bilayered and free-configurations for piezoelectric thicknesses above $200 \mu \mathrm{m}$.

While both, PZT and PVDF based free PMP configurations double the bilayer magnetoelectric coefficient in the whole piezoelectric thickness range, the clamped configuration introduces some differences. When clamped, the PZT PMP configuration reduces ME performance when increasing the piezoelectric layer thickness. On the contrary, PVDF clamped configuration increases the ME response with increasing piezoelectric thickness, starting with a near-to zero response at $10 \mu \mathrm{m}$, but increasing the bilayer ME response for piezoelectric layer thickness of $20 \mu \mathrm{m}$ and even being larger that the free configuration for piezoelectric thickness above $50 \mu \mathrm{m}$ on each side of the Vitrovac layer.

This effect, as well as the exceptions where PVDF laminate's performance depended on the magnetostrictive thickness of the composite, can be explained by the results presented in Figure 6. Figure 6 shows that the electric potential in PZT laminates present a laminar electric potential distribution on the polarization direction and homogeneous displacement field in the deformation 
direction (y) through the thickness of the material. PVDF configurations, on the other hand, shows a non-homogeneous electric potential distribution. MPM-clamped configurations present an even less homogeneous electric potential distribution than the clamped ones, while PMP-clamped configurations present the opposite trend. These facts can be explained by the displacement field in the deformation direction that was 0.1 times the order of the free configuration for the MPM configuration, while presenting twice for the PMP counterpart. Therefore, obtaining higher reponse for clamped PMP configurations than for the free ones (for magnetostrictive thickness of $25 \mu \mathrm{m}$ ) and lower for the clamped MPM configurations than the free ones.

a)

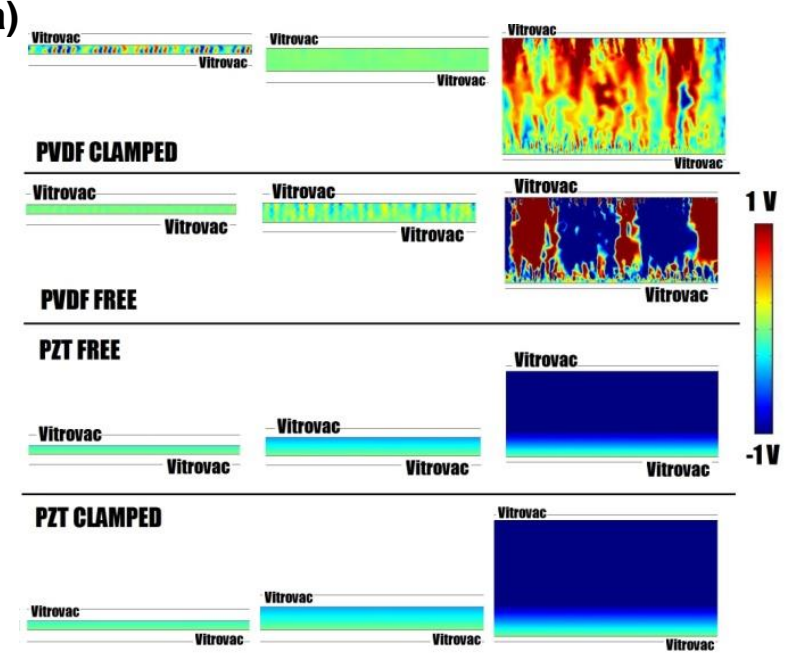

c)

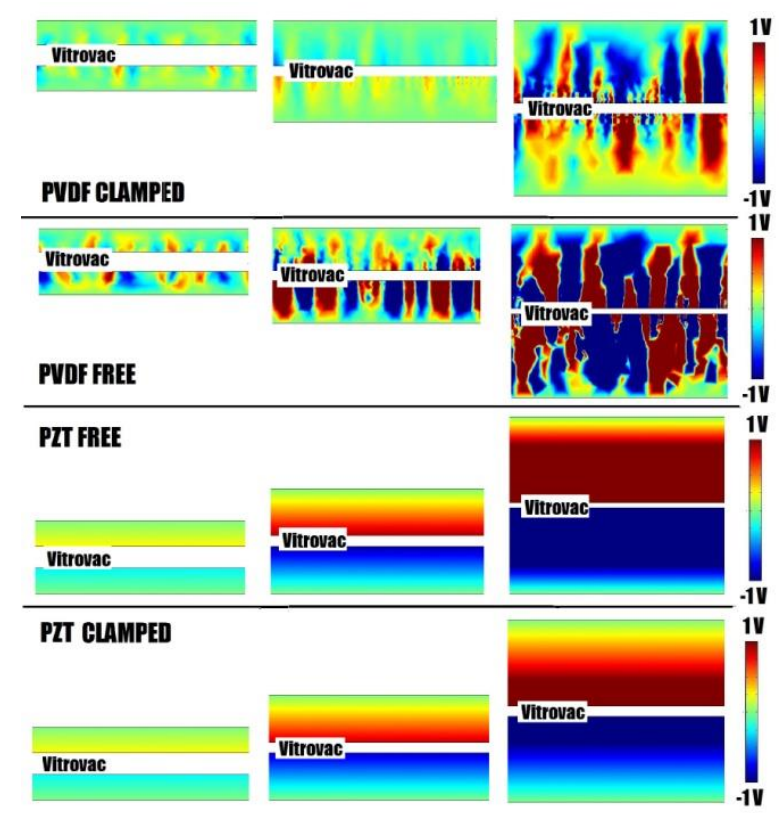

b)

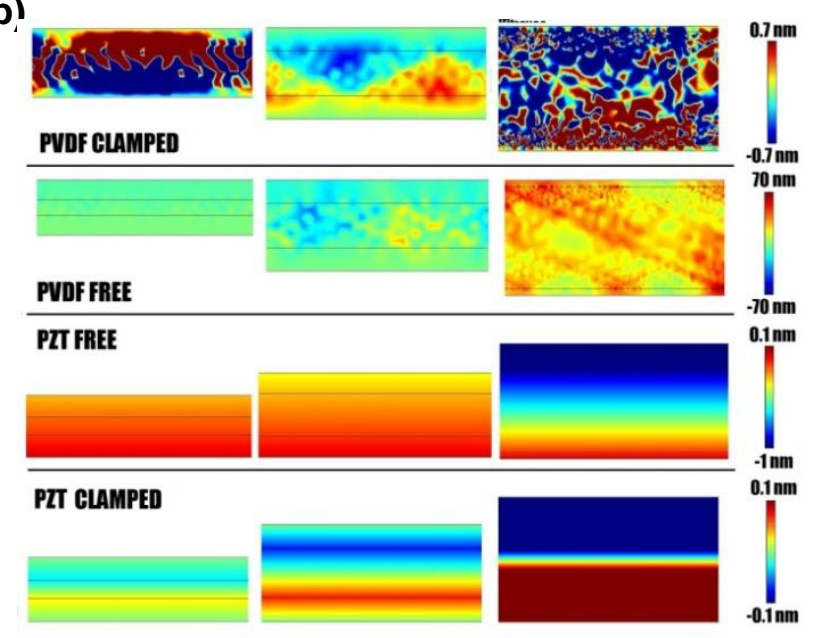

d)

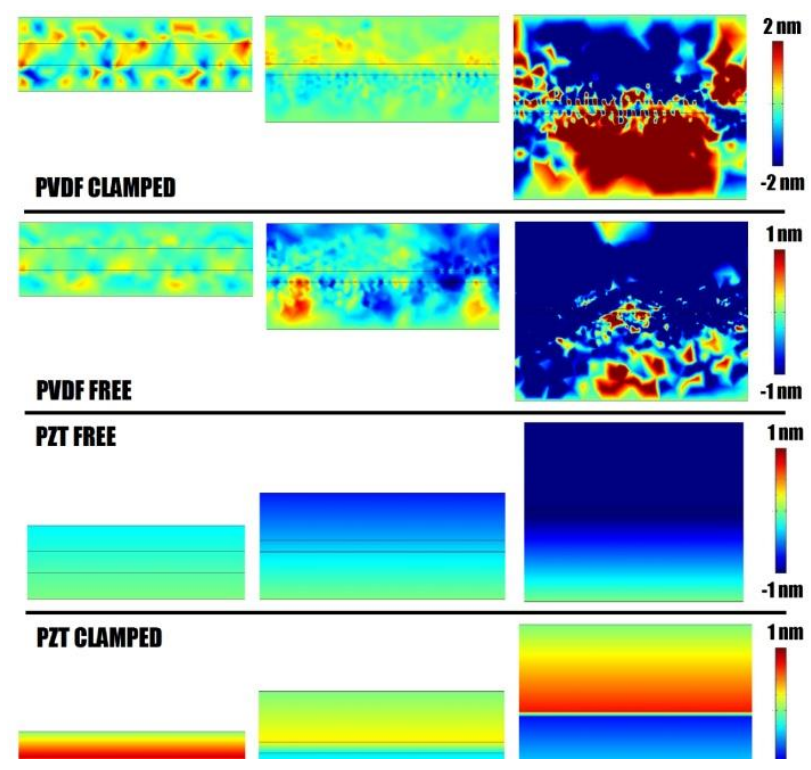

Figure 6: Representation of the electric potential distribution and vertical displacement of trilayered configurations. Figures (a) and (b) represent the electric potential distribution and displacement along the deformation direction of MPM laminates with d_ms $=25 \mu \mathrm{m}$ and d_pzo of

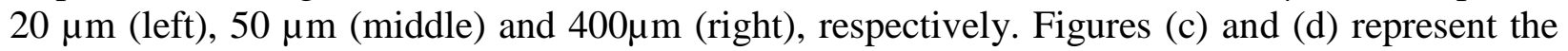


same information of PMP laminates with d_ms=25 $\mu \mathrm{m}$ and d_pzo of $30 \mu \mathrm{m}$ (left), $110 \mu \mathrm{m}$ (middle) and $400 \mu \mathrm{m}$ (right), respectively.

Ryu et al. [23] established the theoretical response for the ME voltage coefficient (dE/dH) of MPM heterostructures as function of the thickness ratio (d_ms/d_pzo), presenting that the output voltage increases while increasing the thickness ratio, saturating above d_ms/d_pzo=10. This trend is corroborated in measurements in [23] Terfenol-D/PZT/Terfenol-D laminates, [18] Metglas/PZT/Metglas configurations, [34] Co/PZT/Co heterostructures and [35] for the ME performance of different thickness ratio values of Terfenol-D/PMN-PT/Terfenol-D laminates. Wong [35] also measured $\boldsymbol{\alpha}_{M E}$ as function of bias magnetic field for PMP laminates based on PMN-PT/Terfenol-D/PMN-PT with three different thickness ratio of 0.4, 1.4 and 2.4. The results showed that the ME performance of the PMP configurations increases with increasing the thickness ratio. Finally, a comparison was stablished between the ME response of MPM and PMP composites, where both configurations rapidly increased their unitary ME behaviour $\left(\boldsymbol{\alpha}_{\text {unit }}\right)$ with increasing the thickness ratio, until reaching saturation. The only difference observed was that MPM configurations reached saturation with a higher slope than PMP configurations, and with a higher ME coefficient (PMP- $\alpha$ in saturation is approximately an $85 \%$ of MPM- $\alpha$ ).

Figure 7 shows a comparison between the ME response of MPM and PMP composites with PZT (a) and PVDF (b) as piezoelectric layers.
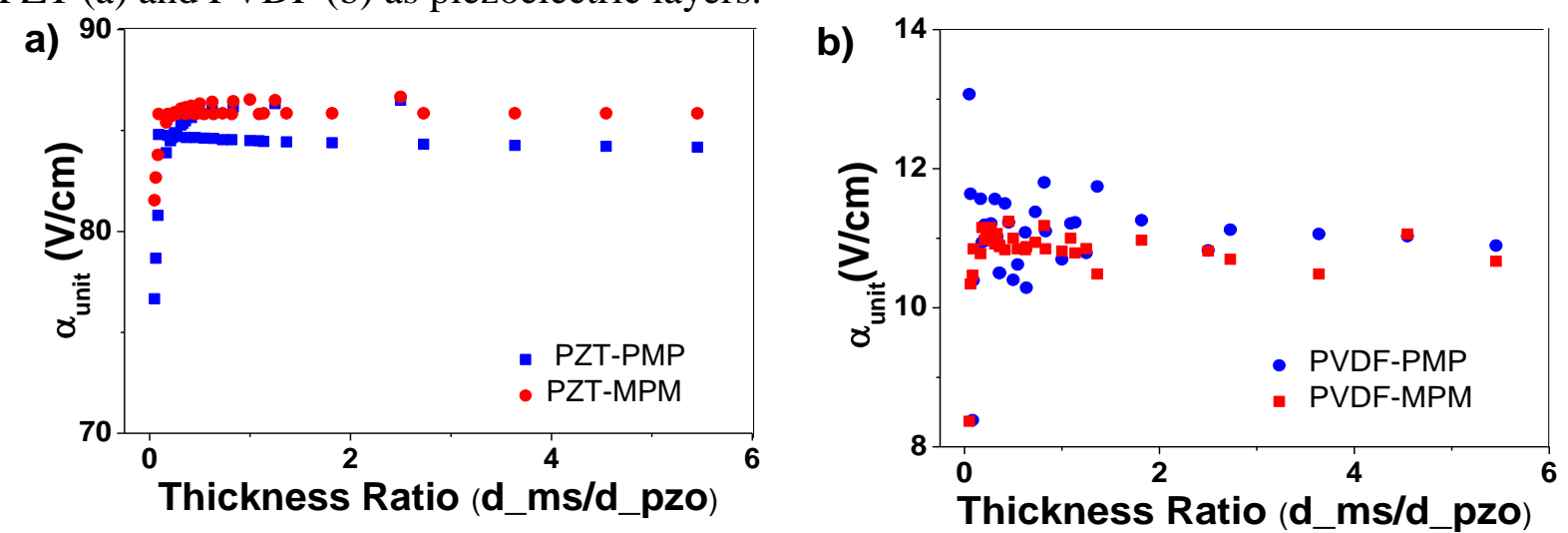

Figure 7: Theoretical ME response for PMP and MPM configurations as a function of magnetostrictive/piezoelectric thickness ratio (d_ms/d_pzt) for PZT-trilayers (a) and PVDFtrilayers (b).

While the trends for PZT configurations confirm the ones established by Ryu et al. [23], they show a much more abrupt slope for MPM configurations. PZT-PMP laminates ME response as a function of thickness ratio shows an increasing ME response for thickness ratio below 0.3, and saturating above this thickness ratio value. PVDF-PMP laminate simulations show a more disordered trend, with a decreasing $\alpha$ for thickness ratios below 0.1 , then increasing until reaching saturation for a thickness ratio of 0.3. This difference is due to the mechanical properties of PVDF, that do not allow to completely transmit deformations when the piezoelectric thickness is too large, therefore decreasing the ME response. 
While the trends presented by Wong[35] are confirmed by PZT MPM and PMP composites simulation results, PVDF MPM and PMP simulations results do not lead to clear tendencies. PZT MPM and PMP simulation results display high-sloped increase of the ME response with increasing thickness ratio, the MPM-configuration reaching saturation for a smaller thickness ratio than the PMP configuration, with a higher ME coefficient in about $2 \mathrm{~V} / \mathrm{cm}$. On the other hand, PVDF MPM and PMP configurations, present curves that match the high-sloped increase of the ME response with increasing thickness ratio and saturating afterwards, but the higher viscoelasticity of PVDF plays an essential role and, after unity of thickness ratio is reached, PMP configurations lead to a better unitary ME response than MPM configurations.

\subsection{Multi-layered composites}

Figure 8 shows the simulation results for M-M and P-P configurations ME response as function of the thickness ratio (d_ms/d_pzo) for PVDF and PZT piezoelectric layers.

PZT M-M and P-P laminates show an enhanced and constant value ME response for either undersized magnetostrictive thicknesses or bulky piezoelectric thicknesses, as presented in Figure 8 (a) and Figure 8 (c). It is shown, that the ME coefficient $\boldsymbol{\alpha}_{\text {unit }}$ increases rapidly while increasing thickness ratio for both configurations, saturating around a thickness ratio of 0.3 and showing therefore a constant response for higher thickness ratios. For high thickness ratios the M-M configuration shows a higher stable $\mathrm{ME}$ value on about $1 \mathrm{~V} / \mathrm{cm}$, showing a slight improvement by the addition of more layers on the M-M configuration. With respect to the P-P configuration, on the other hand, the highest ME coefficient is presented by the 5-Layer P-P configuration. For both $\mathrm{M}-\mathrm{M}$ and P-P configurations and low thickness ratio (below 0.2, meaning high piezoelectric thicknesses and low magnetostrictive thicknesses), the highest number of layers present to introduce an increase on the ME performance of the material is XXXXX.
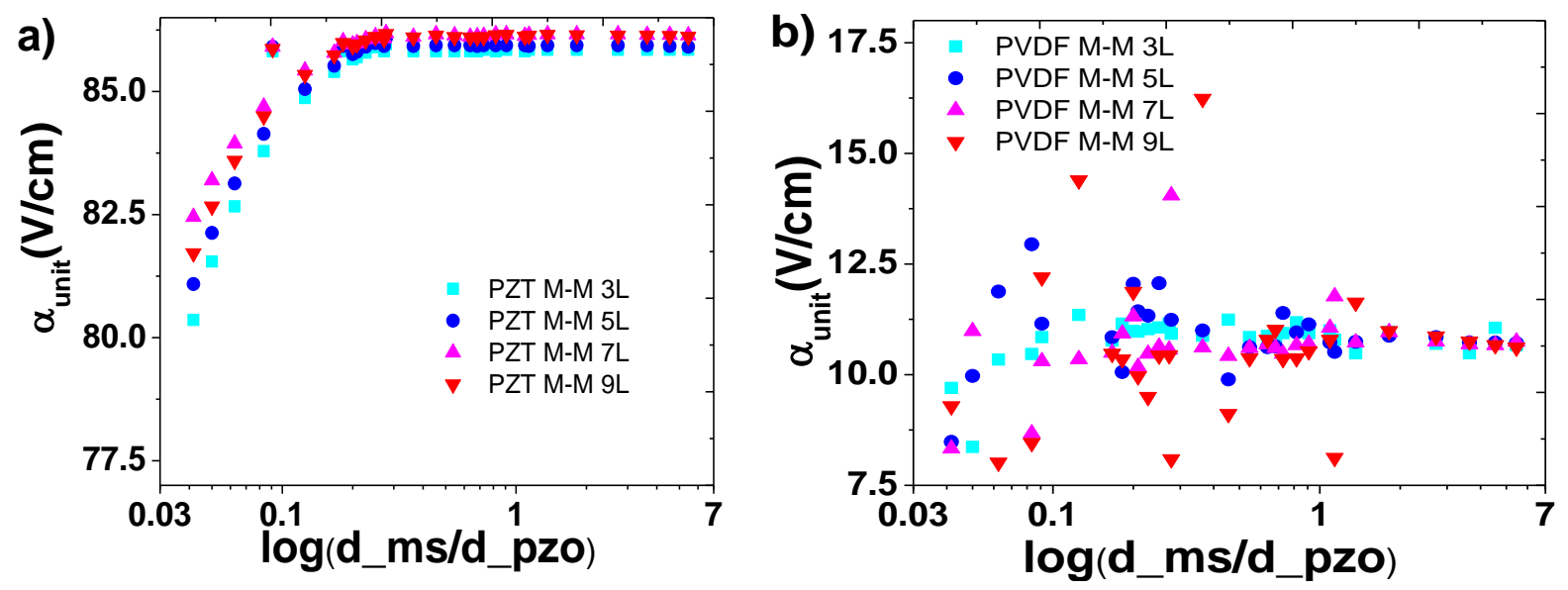

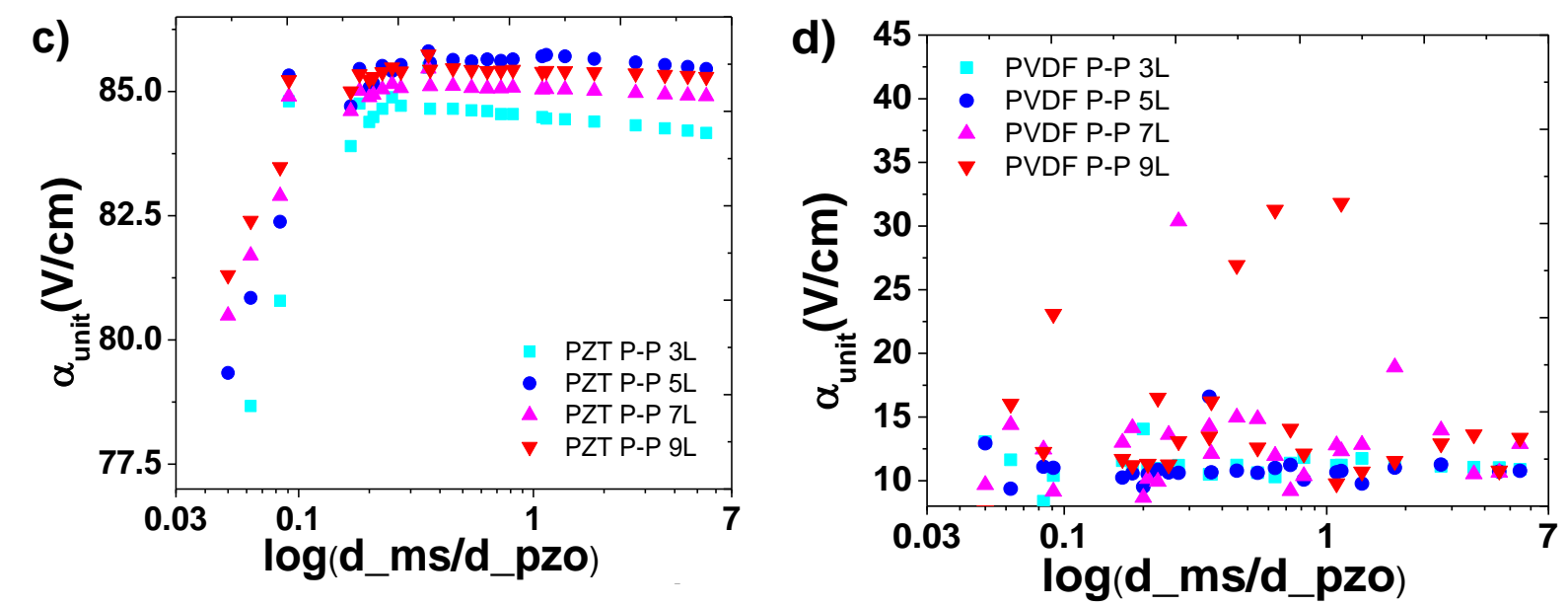

Figure 8: Simulation results for MM and PP multilayered configurations (L indicating the number of layers) as a function of the thickness ratio (d_ms/d_pzo), for PZT M-M (a), PVDF M-M (b), PZT P-P (c) nas PVDF P-P configurations.

PVDF M-M and P-P configurations also preserve ME drifts over magnetostrictive thickness, in particular for low-ordered multilayer configurations (below 5 layers). For higher number of layers, the ME response is governed by the malleability and semicrystalline behavior of the piezoelectric layer, therefore including high number of nonlinearities (peaks). This fact indicates a better ME response when avoiding damping and magnetic losses in the coupling factor. When a high number of layers are involved in the composition of the laminate, the mechanical effects of the viscoelastic PVDF lead to more heterogeneous patterns of ME coefficient. Although preserving the trend reported in [35] for low layer number in PVDF P-P multilayered configurations, the introduction of higher number of PVDF layers in the composite induces a nonlinear behavior. Those nonlinearities present themselves as peak values for specific thickness ratios, establishing improvements when damping or magnetic losses are not considered. It can be also noticed, that the trend of PVDF-PMP $\boldsymbol{\alpha}_{M E}$ versus thickness ratio is preserved for the 5-layered P-P configuration, obtaining high ME response values for thickness ratios below 0.1 , then decreasing quickly to increase again and saturating for thickness ratios above 0.6, even achieving similar ME performance values.

Figure 9 shows simulation results for particular cases of symmetric multilayerd M-M and PP configurations with unity of thickness ratio (d_ms=d_pzo) for thicknesses of $25 \mu \mathrm{m}(25 / 25)$ and $110 \mu \mathrm{m}(110 / 110)$.

PZT M-M and P-P configurations present higher ME coefficient for lower thicknesses, with higher values for $\mathrm{M}-\mathrm{M}$ than $\mathrm{P}-\mathrm{P}$ configurations. M-M configurations show a uniform ME coefficient as a function of the number of layers, with higher values than the bilayer composite. P$\mathrm{P}$ configurations, on the other hand, show the highest ME response for the 5-Layer configuration, reaching the value of the M-M configuration for the thin composite (25/25 P-P). 

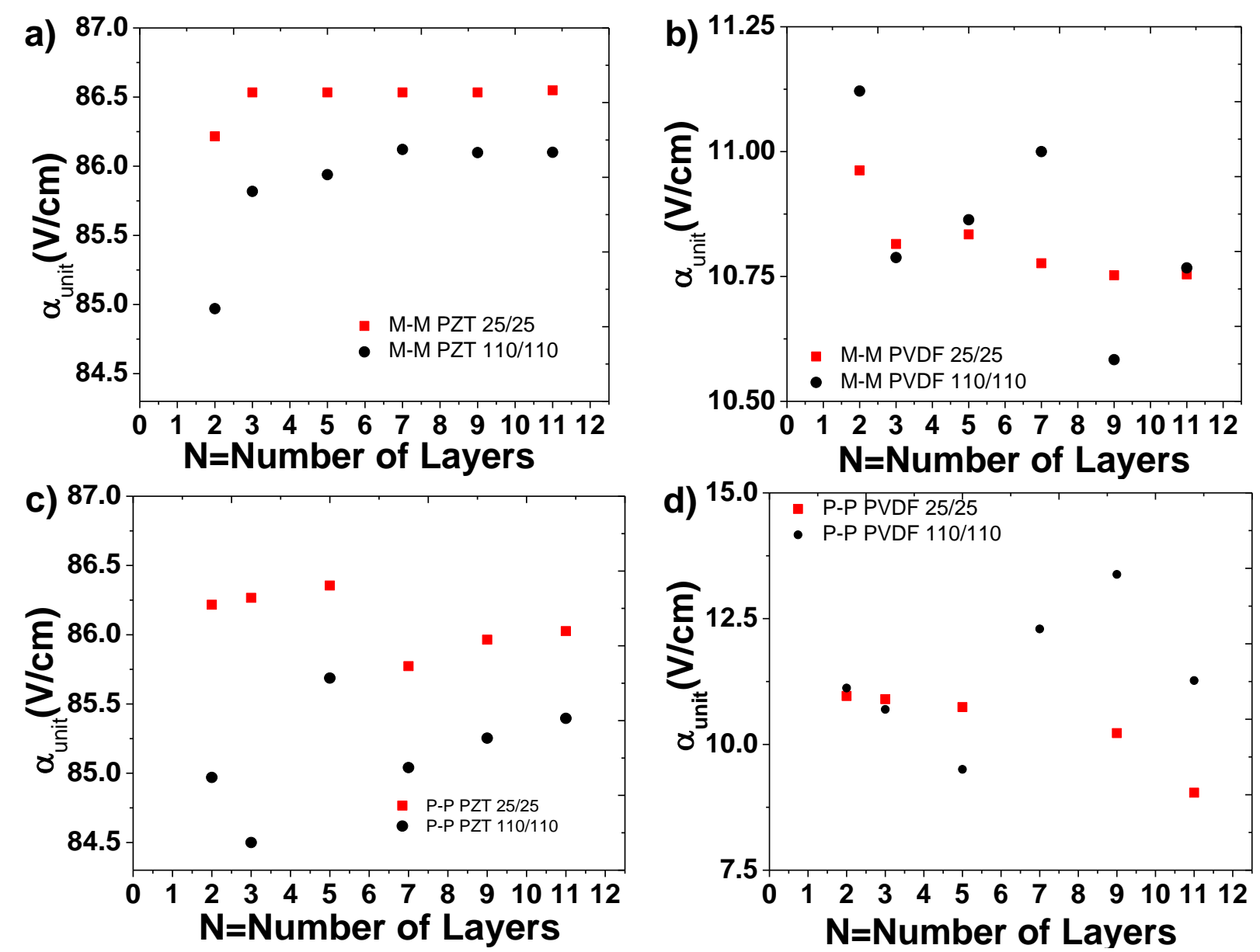

Figure 9: Simulation results for symmetric M-M and P-P multilayered configurations as function of the number of layers, $\mathrm{N}$, when magnetostrictive and piezoelectric layers have the same thickness, of 25 and $110 \mu \mathrm{m}$, for M-M PZT/Vitrovac (a), M-M PVDF/Vitrovac (b), P-P PZT/Vitrovac (c), and P-P PVDF/Vitrovac (d).

PVDF M-M and P-P configurations are influenced by the viscoelasticity of the electroactive polymer. M-M configurations show clear trends of decreasing ME coefficient with increasing number of layers, presenting a maximum performance for the bilayer structure. P-P configurations show no stable behavior, exhibiting a ME coefficient 4 times higher than any other configuration for the $25 \mu \mathrm{m}-7$ layer composite, of $48 \mathrm{~V} / \mathrm{cm}$. Figure 10 shows the electric potential distribution for 3, 5, 7 and 11 layered ME composites with $25 \mu \mathrm{m}$ layer thickness. It is observed in (c) that the top piezoelectric layer of the 7-layerd composite is producing under deformation a series of dipolarities that are at the origin of this enhanced $\mathrm{ME}$ response. Beside this isolated enhanced ME responses of the 7-layered 25/25 ME composites, results show better performance for P-P configurations of 110/110 and 7 or 9 layers, improving the ME response for the latter around 3 $\mathrm{V} / \mathrm{cm}$ in comparison with the bilayer configuration. 


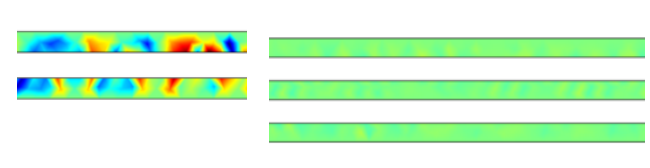

a)

b)

c)

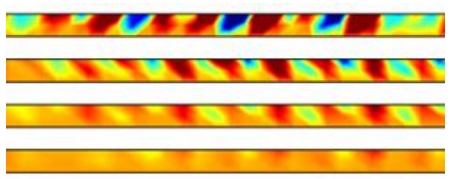

d)
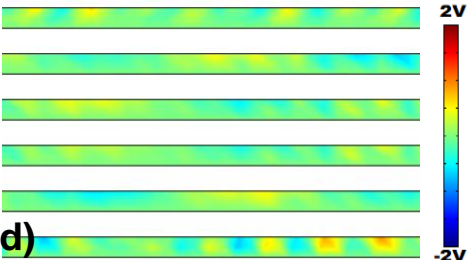

Figure 10: Electric potential distribution of P-P configurations with 3 (a), 5 (b), 7 (c) and 11 (d) layers with a colour range between $2 \mathrm{~V}$ (dark red) and $-2 \mathrm{~V}$ (dark blue)

\subsection{Constant ME thickness multilayer experiment}

Srinivasan et al. [37] evaluated the ME effect in bilayers and multilayers of thick-film structures composed of (LSMO)-PZT and (LCMO)-PZT, in which samples were fabricated with an equal number of piezoelectric and magnetostrictive layers, between 2 and 8, maintaining constant the total thickness, $\mathrm{D}_{\mathrm{C}}$, of the device and the thickness of both magnetostrictive and piezoelectric phases. This study concluded that transverse ME voltage is lower in multilayers compared to bilayers, and that the relation is linear and proportional to the number of layers, $\mathrm{N}$. Simulation results for PZT and PVDF laminates are shown in Figure 11 (a) and (b), respectively, with corresponding electric potential distribution Figure 11 (c).
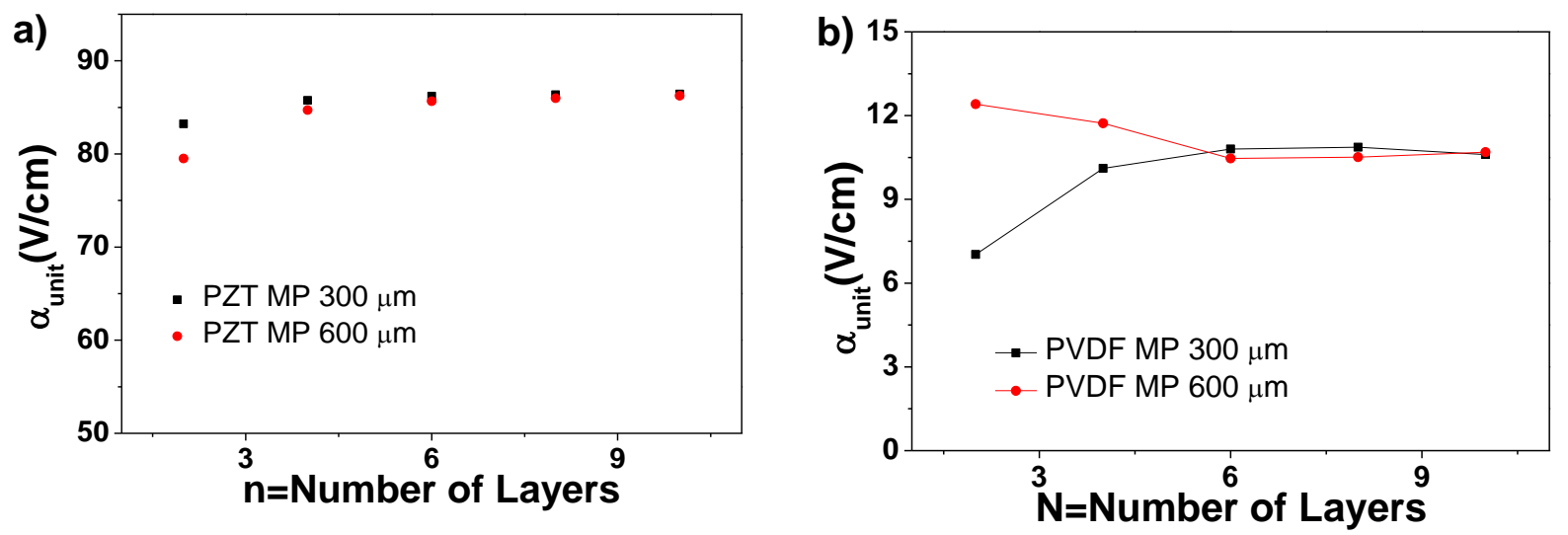

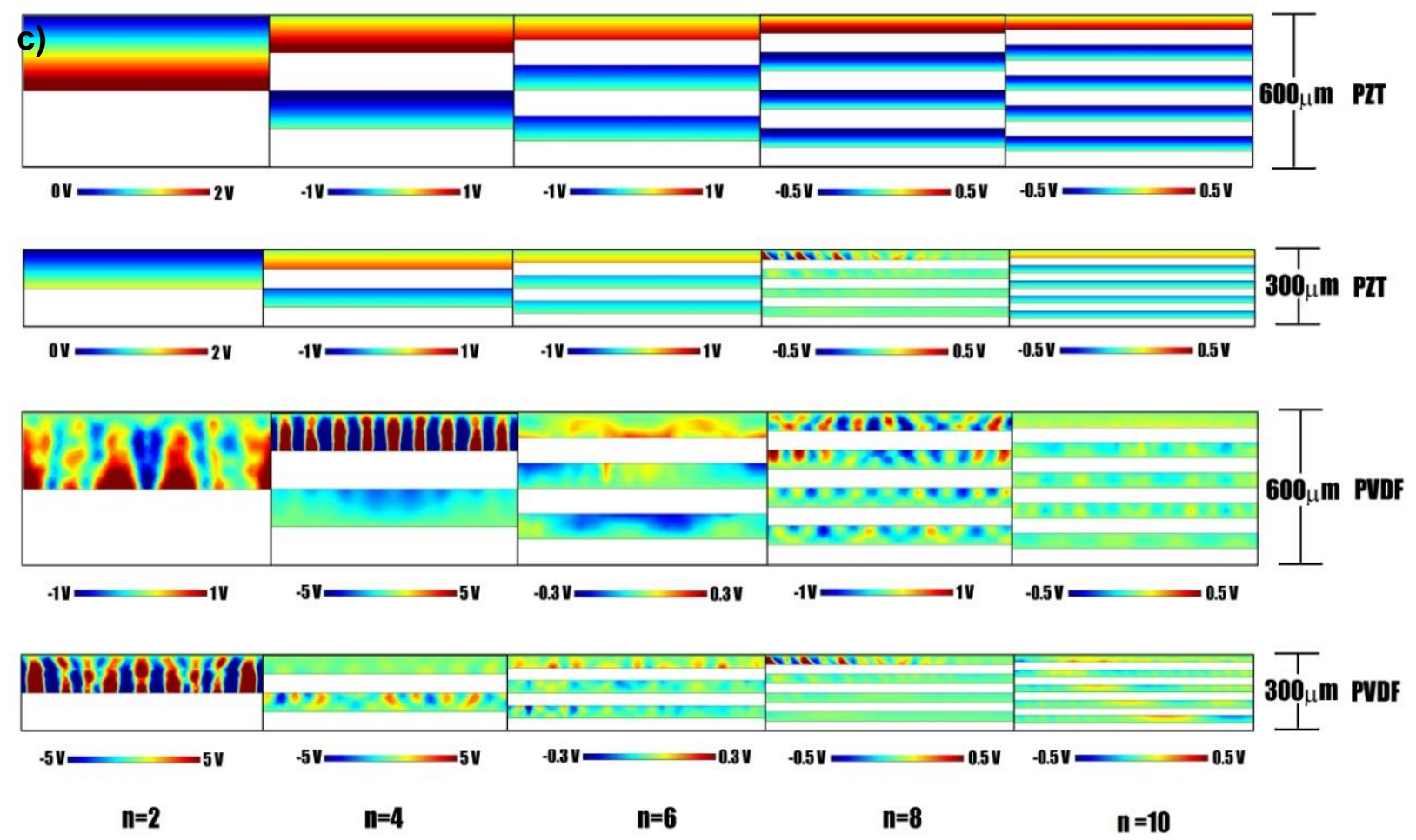

Figure 11: Simulations of peak ME voltage coefficients for PZT M-P (a) and PVDF M-P (b) multilayers as a function of the numbers of layers, N. In figure (c) it is shown the electric potential distribution for FEM simulations with PZT (top) and PVDF (bottom) M-P multilayers, with 2 to 10 layers, with the cases of $600 \mu \mathrm{m}$ and $300 \mu \mathrm{m}$ of total thickness.

PZT M-P laminates show an increase in the ME response when divided into multilayers for both total thicknesses, $D_{c}$, of 300 and $600 \mu \mathrm{m}$, a different trend that the one reported in [37]. The differences on the simulations based on PZT as piezoelectric layers can be related to the lack of consideration, in the present simulations, of structural damping and magnetic losses, which may play a relevant role when more layers are introduced. Although the $300 \mu \mathrm{m}$-thick PVDF M-P laminate shows the same results as the PZT M-P simulation trends, the PVDF multilayer of total thickness of $600 \mu \mathrm{m}$ follows Srinivasan's et al. results, showing a slightly improved ME unitary performance as a lower number of layers is involved. The electric potential distribution in Figure 11 (c) shows the homogeneous pattern in the PZT configurations, and the inhomogeneous patterns for PVDF M-P laminates. While the thinner PVDF M-P ME composites already presented a dipolar inhomogeneous pattern in the bilayer configuration, the composites with total thickness of $600 \mu \mathrm{m}$, only show this effect in the top of the piezoelectric layer for $\mathrm{N}=6$ or lower. In these cases, the poles appeared to be bigger and therefore the ME performance is enhanced, even showing an approximately homogeneous electric potential distribution pattern for at least one of the piezoelectric internal layers for $\mathrm{N}=4$ and $\mathrm{N}=6$. 
Figure 12 presents the results of the ME unitary coefficient, $\boldsymbol{\alpha}_{\boldsymbol{u} \text { init, }}$ as function of the configuration (M-M, P-P or MP) and de number of piezoelectric layers in the composite, when the total thickness is held constant at 300 and $600 \mu \mathrm{m}$.
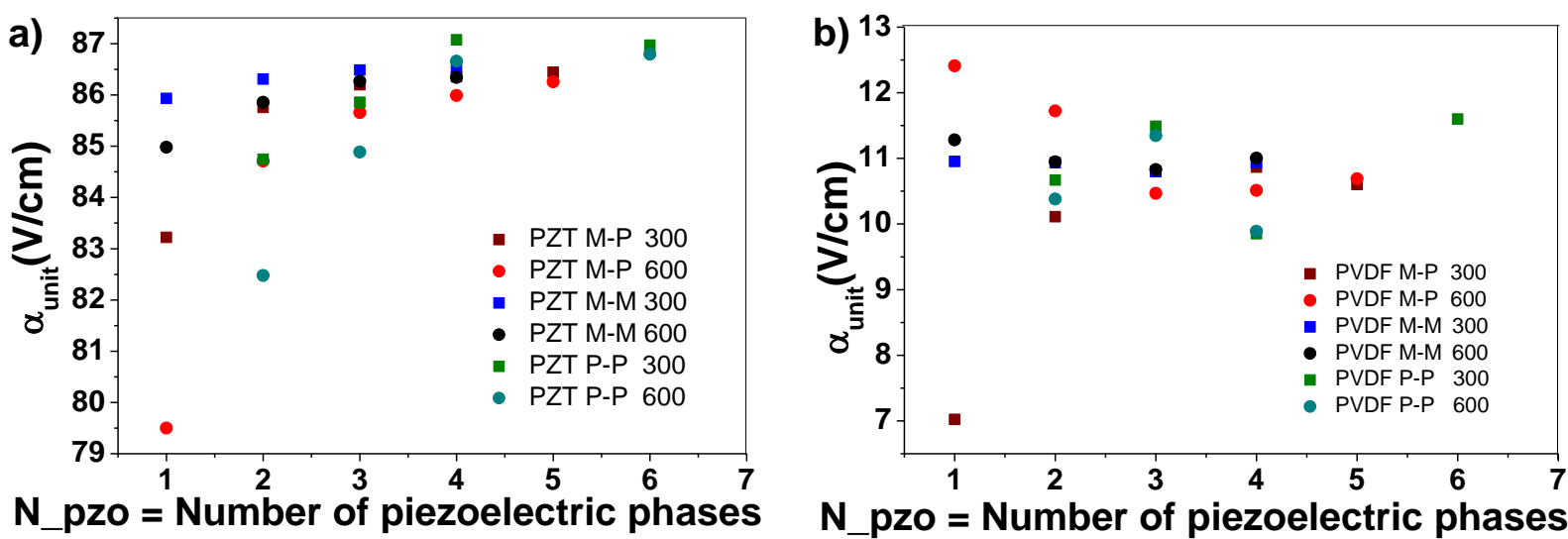

Figure 12: Unitary ME coefficient for PZT MP, M-M and P-P (a) and PVDF MP, M-M and P-P (b) constant thickness composites with a total thickness of $300 \mu \mathrm{m}$ and $600 \mu \mathrm{m}$ as function of the number of piezoelectric layers, N_pzo.

PZT M-M laminates show increasing ME response with increasing the number of piezoelectric layers (N_pzo), with a higher $\boldsymbol{\alpha}_{M E}$ for thinner layers and low amount of layers but reaching similar results for more than 7 layers composites (N_pzo>3). Those results are similar to ones for MPM laminates. PZT P-P experiments also show higher $\boldsymbol{\alpha}_{\text {unit }}$ for thinner layers, taking approximately a constant value. In general, all PZT configurations show the trend of increasing $\mathrm{ME}$ response with increasing the number of piezoelectric layers, obtaining an enhanced response for M-M configurations with less than 4 piezoelectric layers, but reaching its maximum value for the P-P configurations with 4 electroactive phases. It is to notice, that these higher values for high number of layers can decrease when considering other effects as coupling parameters for bonding properties or magnetic losses. It can be concluded than in M-P and P-P configurations the role of the composite's thickness plays a larger role than in M-M configurations. These showing more stable values, with a maximal difference of $1 \mathrm{~V} / \mathrm{cm}$ for 1 piezoelectric layer, for low number of piezoelectric layers.

Based on the elasticity and semicrystallinity of the piezoelectric polymer, PVDF M-M configurations show larger $\alpha$-values for low-layered composites with higher thickness, and with more stable unitary $\alpha$ over different number of layers (with an almost constant value of unitary $\boldsymbol{\alpha}$, of approx. 10.7-10.9 V/cm ). For 5 or more layers (N_pzo>2), PVDF M-M experiments show a clear trend of maintaining $\alpha$-values independently of the total thickness of the composite. As obtained for the PVDF M-P constant thickness configurations, PVDF M-M composites performed better as a bulk-bilayered composite than in a thinner multi-layered ones. Also considering the results obtained by Srinivasan et al. [36] and considering damping, bonding and magnetic losses, it may be concluded that thicker bilayered PVDF composites will result in an enhanced performance over multilayered thinner ones. 
M-P configurations, on the other hand, present high ME response for a low number of layers and higher thicknesses, and an increasing ME response of thin composites with the amount of layers. While the bilayered M-P $600 \mu \mathrm{m}$ configuration shows the highest $\boldsymbol{\alpha}$, of approx. $12.4 \mathrm{~V} / \mathrm{cm}$, the bilayered M-P $300 \mu \mathrm{m}$ shows the lowest value of $7 \mathrm{~V} / \mathrm{cm}$. P-P configurations, on the other hand, show no clear trends, as the highest achieved value is established by the P-P $600 \mu \mathrm{m}$ with more than double $(27 \mathrm{~V} / \mathrm{cm})$ the highest M-P ME coefficient value. This confirms also, that P-P configurations may not presented clear trends and that the elasticity and morphology of PVDF has a larger influence in this configuration.

\section{Conclusions}

A structural simulation model based on Finite Element Methods (FEM) for magnetoelectric (ME) composites was developed in order to establish the influence of the mechanical properties and morphology of the different layers in the ME response. Ceramic (PZT) and polymer (PVDF) based magnetostrictive multilayered composites were analyzed according to their configuration, thickness and number of layers. This comparison, allows to establish the influence of the structural properties of the piezoelectric layers in the ME coupling, leading to a better understanding of composites behavior.

$\mathrm{XX}$

PZT composites simulations allowed to obtain clear trends of enhanced composites, for bilayers with thinner piezoelectric thicknesses (below $200 \mu \mathrm{m}$ ) and magnetostrictive thicknesses above 50-60 $\mu \mathrm{m}$ for clamped configurations. Good agreement was established when comparing with the trends reported experimentally in the literature for magnetostrictive to piezoelectric thickness ratio of multilayers, presenting a curve that rapidly increases their $\boldsymbol{\alpha}_{\text {unit }}$ with increasing thickness ratio until $n=0.3$, when saturation is reached. The difference with $M-M$ configurations is that they reached saturation with a higher slope than P-P configurations, to a higher value, therefore presenting an optimal configuration for multilayers with external magnetostrictive phases (M-M configurations), with thickness ratio above 0.2 (being the thickness of the magnetostrictive phase higher than 5 times the electroactive phase's thickness), of about $86.7 \mathrm{~V} / \mathrm{cm}$.

PVDF bi-layers, on the other hand, exhibit a constant $\alpha_{\mathrm{ME}}$ values of $10.71 \mathrm{~V} / \mathrm{cm}$, for polymer layers below $110 \mu \mathrm{m}$, and then some higher and lower $\boldsymbol{\alpha}_{\mathrm{ME}}$ values. This established a clear influence of the semicrystalline nature and viscoelasticity of PVDF in this heterogeneous ME behaviour with isolated peak values. These effects introduce difficulties in establishing trends on the influence of thicknesses and configuration on the ME coefficient of the multi-layered composites. It may be established that M-M configurations show more stable behaviour and trends over different number of layers, while P-P configurations present regions with peaks that went off the expected trend. It can be also stablished that M-P configurations are severely dependent on the composite's thickness. For configuration with less than 3 piezoelectric layers trends show no particular influence of magnetostrictive thickness over the ME performance of free PVDF multilayered configurations, and a slight decrease of $\alpha_{\mathrm{ME}}$ above piezoelectric thicknesses of $150 \mu \mathrm{m}$. Clamped configurations, on the other hand, show a ME performance that is dependent of magnetostrictive and piezoelectric thicknesses. For free configurations, thin PVDF thicknesses improve the ME response of the composite, with the possibility of the incorporation of more than one layer to enhance the performance of the structure.

As PZT-based ME composites present clear trends, it appears necessary to incorporate bonding effects, damping and magnetic losses to the reported simulations. Bonding and damping 
effects will be reinforced with the incorporation of more layers in the composite and the present structural model should be improved by incorporating these variables into simulations. With the incorporation of damping and bonding effects, multi-layer structural simulation results would be much more effective and would allow a better understanding of the peak variations presented by P-P configuration. Also, this analysis suggested that every configuration of PVDF-laminate has to be carefully analyzed within the mounting conditions. Resources should be devoted into establishing a nonlinear magnetostrictive model that could be coupled to this structural model, in order to obtain more information about the magnetostrictive performance of the material, and to compare different options of magnetostrictive materials. PVDF's biocompatibility, elasticity and malleability, makes it suitable for a high number of technological applications.

\section{Acknowledgements}

The authors thank the Erasmus Mundus VECCEU scholarship and FCT- Fundação para a Ciência e Tecnologia- for financial support in the framework of the Strategic Funding UID/FIS/04650/2019 and under projects PTDC/BTM-MAT/28237/2017 and PTDC/EMDEMD/28159/2017. The authors acknowledges funding by the Spanish Ministry of Economy and Competitiveness (MINECO) through the project MAT2016-76039-C4-3-R (AEI/FEDER, UE) (including the FEDER financial support) and and from the Basque Government Industry and Education Departments under the ELKARTEK, HAZITEK and PIBA (PIBA-2018-06) programs is also acknowledged.

\section{REFERENCES}

1. Jing, W.Q. and F. Fang, A flexible multiferroic composite with high self-biased magnetoelectric coupling. Composites Science and Technology, 2017. 153: p. 145-150.

2. Fuentes, M.E., et al., Meso- and nano- magnetoelectricity : a review. Revista Mexicana de Física, enero, 2007. 53(1): p. 21-29.

3. $\mathrm{Li}, \mathrm{Y}$., et al., Fe<inf $>3</$ inf $>0<$ inf $>4</$ inf $>$ decorated graphene/poly(vinylidene fluoride) nanocomposites with high dielectric constant and low dielectric loss. Composites Science and Technology, 2019. 171: p. 152-161.

4. Eerenstein, W., N.D. Mathur, and J.F. Scott, Multiferroic and magnetoelectric materials. Nature, 2006. 442(7104): p. 759-765.

5. Martins, P. and S. Lanceros-Méndez, Polymer-based magnetoelectric materials. Advanced Functional Materials, 2013. 23(27): p. 3371-3385.

6. Ramesh, R. and N.A. Spaldin, Multiferroics: Progress and prospects in thin films. Nature Materials, 2007. 6(1): p. 21-29.

7. Lehmann Fernández, C.S., et al., Theoretical design of high-performance polymer-based magnetoelectric of fibrilar structures. Composites Science and Technology, 2018. 155: p. 126136.

8. Lehmann Fernández, C.S., et al., Evaluation and optimization of the magnetoelectric response of CoFe $<$ inf $>2</$ inf $>0<$ inf $>4</$ inf $>/$ poly(vinylidene fluoride) composite spheres by computer simulation. Composites Science and Technology, 2017. 146: p. 119-130.

9. Zhai, J., et al., Magnetoelectric laminate composites: An overview. Journal of the American Ceramic Society, 2008. 91(2): p. 351-358. 
10. Gutiérrez, J., et al., Metallic glass/PVDF magnetoelectric laminates for resonant sensors and actuators: A review. Sensors (Switzerland), 2017. 17(6).

11. Belouadah, R., et al., Enhancement of the magnetoelectric response of polyurethane polymer / piezoceramic bi-layer materials. Sensors and Actuators, A: Physical, 2019. 299.

12. Maceiras, A., et al., High-temperature polymer based magnetoelectric nanocomposites. European Polymer Journal, 2015. 64: p. 224-228.

13. Jin, J., et al., Multiferroic polymer composites with greatly enhanced magnetoelectric effect under a low magnetic bias. Advanced Materials, 2011. 23(33): p. 3853-3858.

14. Reis, S., et al., Optimized anisotropic magnetoelectric response of Fe61.6Co16.4Si10.8B11.2/PVDF/Fe61.6Co16.4Si10.8B11.2 laminates for AC/DC magnetic field sensing. Smart Materials and Structures, 2016. 25(5).

15. Martins, P., A.C. Lopes, and S. Lanceros-Mendez, Electroactive phases of poly(vinylidene fluoride): Determination, processing and applications. Progress in Polymer Science, 2014. 39(4): p. 683-706.

16. Silva, M., et al., Optimization of the magnetoelectric response of poly(vinylidene fluoride)/epoxy/vitrovac laminates. ACS Applied Materials and Interfaces, 2013. 5(21): p. 10912-10919.

17. Lin, B., et al., Enhanced dielectric permittivity in surface-modified graphene/PVDF composites prepared by an electrospinning-hot pressing method. Composites Science and Technology, 2019. 172: p. 58-65.

18. Fang, F., C. Zhao, and W. Yang, Thickness effects on magnetoelectric coupling for Metglas/PZT/Metglas laminates. Science China: Physics, Mechanics and Astronomy, 2011. 54(4): p. 581-585.

19. Lin, Y., et al., Giant magnetoelectric effect in multiferroic laminated composites. Physical Review B - Condensed Matter and Materials Physics, 2005. 72(1).

20. Nan, C.W., et al., Large magnetoelectric response in multiferroic polymer-based composites. Physical Review B - Condensed Matter and Materials Physics, 2005. 71(1).

21. Ryu, J., et al., Magnetoelectric properties in piezoelectric and magnetostrictive laminate composites. Japanese Journal of Applied Physics, Part 1: Regular Papers and Short Notes and Review Papers, 2001. 40(8): p. 4948-4951.

22. Ryu, J., et al., Magnetoelectric effect in composites of magnetostrictive and piezoelectric materials. Journal of Electroceramics, 2002. 8(2): p. 107-119.

23. Ryu, J., et al., Effect of the Magnetostrictive Layer on Magnetoelectric Properties in Lead Zirconate Titanate/Terfenol-D Laminate Composites. Journal of the American Ceramic Society, 2001. 84(3-12): p. 2905-2908.

24. Zhang, Y., et al., Optimizing the dielectric energy storage performance in P(VDF-HFP) nanocomposite by modulating the diameter of PZT nanofibers prepared via electrospinning. Composites Science and Technology, 2019. 184.

25. Olabi, A.G. and A. Grunwald, Design and application of magnetostrictive materials. Materials and Design, 2008. 29(2): p. 469-483.

26. Pan, E. and R. Wang, Effects of geometric size and mechanical boundary conditions on magnetoelectric coupling in multiferroic composites. Journal of Physics D: Applied Physics, 2009. 42(24).

27. Fonteyn, K., et al., FEM for directly coupled magneto-mechanical phenomena in electrical machines. IEEE Transactions on Magnetics, 2010. 46(8): p. 2923-2926.

28. Li, W. and H.J. Shen, A refined layerwise finite element modeling of delaminated composite laminates with piezoelectric layers. Thin-Walled Structures, 2018. 131: p. 792-804. 
29. Nechibvute, A., A. Chawanda, and P. Luhanga, Finite Element Modeling of a Piezoelectric Composite Beam and Comparative Performance Study of Piezoelectric Materials for Voltage Generation. ISRN Materials Science, 2012. 2012: p. 11.

30. Zadov, B., et al., Modeling of small DC magnetic field response in trilayer magnetoelectric laminate composites. Advances in Condensed Matter Physics, 2012. 2012.

31. Babu, S.N., T. Bhimasankaram, and S.V. Suryanarayana, Magnetoelectric effect in metal-PZT laminates. Bulletin of Materials Science, 2005. 28(5): p. 419-422.

32. Nan, C.W., et al., Multiferroic magnetoelectric composites: Historical perspective, status, and future directions. Journal of Applied Physics, 2008. 103(3).

33. Esterly, D.M., Manufacturing of Poly(vinylidene fluoride) and Evaluation of its Mechanical Properties. Virginia Tech, 2002. Collections Masters Theses

34. Stognij, A.I., et al., Effect of cobalt layer thickness on the magnetoelectric properties of Co/PbZr $<$ inf $>0.45</$ inf $>$ Ti $<$ inf $>0.55</$ inf $>0<$ inf $>3</$ inf $>/$ Co heterostructures. Inorganic Materials, 2013. 49(10): p. 1011-1014.

35. Wong, W.-y., Studies on the direct and converse magnetoelectric effects in laminated composites. The Hong Kong Polytechnic University, 2008. Thesis.

36. Srinivasan, G., et al., Giant magnetoelectric effects in layered composites of nickel zinc ferrite and lead zirconate titanate. Solid State Communications, 2002. 124(10-11): p. 373-378.

37. Srinivasan, G., et al., Magnetoelectric effects in bilayers and multilayers of magnetostrictive and piezoelectric perovskite oxides. Physical Review B - Condensed Matter and Materials Physics, 2002. 65(13): p. 1344021-1344027. 